(Aus dem physiologischen Institute der k. k. böhm. Universität in Prag.)

\title{
Der physiologische Protoplasmastoffwechsel und die Purinbildung.
}

Von

Dr. F. Mareš,

Professor der Physiologie.

Zur Erforschung der bestimmten physiologischen Tätigkeiten zugrunde liegenden Stoffwechselvorgänge dient in erster Linie das Verhältnis der Ausscheidung bestimmter Stoffwechselprodukte zu diesen Tätickkeiten. Unter diesen Produkten kommt der Harnsäure und den Purinkörpern überhaupt eine besondere Bedeutung zu. Dann erstens entsteht die Harnsäure, im Gegensatze zum Harnstoffe, aus stofflichen Änderungen im tätigen Zellprotoplasma selbst dies babe ich aus meinen im Jahre 1887 durchgeführten Untersuchungen zuerst erschlossen. Und zweitens sind es insbesondere die Nukleinsubstanzen des Protoplasmas, von welchen die Harnsäure und die Purinkörper des Harnes herrühren; darauf weist der von Kossel und von Horbaczewski nachgewiesene chemische $\mathrm{Zu}$ sammenhang der Alloxurkörper und der Harnsäure mit den $\mathrm{Nu}-$ kleinen hin.

Meine Untersuchungen, durch welche der Ursprung der Harnsäure im tätigen Zellprotoplasma nachgewiesen wurde, im Gegensatze zum Harnstoffe, der von dem verdauten Nahrungseiweiss herrührt, sind zu jener Zeit durchgefuhrt worden, wo man noch ziemlich allgemein die Harnsäure als eine minder oxydierte Vorstufe des Harnstoffes direkt aus dem Nahrungseiweiss herleitete, da man ja auch immer nach reichlicher Eiweiss-(Fleisch-)Nahrung eine anscheinend parallele Harnsäure- sowie Harnstoffvermehrung beobachtet hat.

Diese Annahme hing vielleicht mit der allgemeinen Vereinfachung der Stoffwechsellehre durch Voit zusammen welche die Lehren Liebig's verdrängte. So hatten Lehmann, Ranke, Meissner, 
im Sinne dieser Lehren, für Harnstoff und Harnsäure verschiedenen Ursprung angenommen. Ranke besonders führte die Harnsäure auf das Hypoxanthin der Milz zurück, deren Anschwellung in einem bestimmten Verdauungsstadium ihm mit Harnsäurevermehrung verbunden zu sein schien. Aber der Zusammenhang zwischen der Harnsäure und den Xanthinkörpern war zu der Zeit, als jch meine Versuche vornahm, ganz problematisch. Kossel begründete eben damals die Kenntnis der Beziehungen der Xanthinkörper zu den Nukleinen; aber die Beziehung der Harnsäure zu diesen Körpern war zweifelhaft, da es nicht gelingen wollte, durch Einnahme derselben Harnsäurevermehrung zu bewirken; so blieb man bei der Annahme, dass die Harnsäure als Vorstufe des Harnstoffes aus dem Nahrungseiweiss herstamme.

Dagegen brachten meine Untersuchungen zuerst den Nachweis, dass die Harnsäureausscheidung beim Menschen ganz andere Verhältnisse zeigt als die Harustoffausscheidung, so dass diese beiden Körper verschiedenen Ursprungs sein dürften, und zwar so, dass der Harnstoff aus dem verdauten und resorbierten Nahrungseiweiss herstammt, die Harnsäure aber aus stofflichen Äpderungen im Zellprotoplasma selbst hervorgeht. Ich dachte zunächst an die Drüsenzellen des Verdauungsapparates, deren durch Eiweisseinnahme angeregte Tätigkeit mit sichtbaren stofflichen Änderungen im Zelleibe, zur Bereitung der Verdauungssäfte, verbunden ist. Ich fand nämlich nach Einnahme von Fleisch eine rasche Vermehrung der Harnsäureausscheidung, deren zeitlicher Verlauf augensebeinlich auf einen $\mathrm{Zu}$ sammenhang mit der Bereitung der Verdauungssekrete hindeutete, während die Vermehrung des Gesamtstickstoffes (Harnstoffes) später, augenscheinlich mit der Resorption des verdauten Nahrungseiweisses, zum Vorschein kam. Ich fand weiter, dass die durch Pilokarpineinwirkung angeregte Drüsentätigkeit tatsächlich mit Harnsäurevermehrung verbunden ist.

Aber diese Untersuchungen fanden zunächst wenig Beachtung. Denn erstens sind dieselben an wenig oder gar nicht zugänglichen Orten ${ }^{1}$ ) veröffentlicht worden, so dass sie zur Kenntnis weiterer Kreise nur durch kurze Referate ${ }^{2}$ ) ohne Belege gelangen konnten. Dann

1) F. Marěs, Sur l'origine de l'acide urique chez l'homme. Arch. Slaves de Biologie t. 3 p. 207-226. 1887. - Sborník lékarský t. 2 p. 1-18. 1888.

2) Zentralbl. f. d. med. Wissensch. 1888 S. 2 (J. M unk). - Zentralbl. f. Physiol. Bd. 1 S. 444. 1888. (F. Röhmann.) 
aber wurde bald die allgemeine Aufmerksamkeit in der Frage der Harnsäure auf die Untersuchungen von Horbaczew ski ${ }^{1}$ 'gerichtet, durch welche die Beziehung der Harnsäure zu den Xanthinkörpern und zu den Nukleinen tatsächlich nachgewiesen worden ist. Hor baczewski schuf dann auch eine eigene Theorie der Harnsäurebildung im Säugetierorganismus, welche auch gleich ziemlich allgemein angenommen wurde. Danach sollte die Harnsäure ausschliesslich aus den Nukleinen der im lebenden Körper nekrotisch zerfallenden Leukocyten entstehen. Als tatsächlicher Grund dieser Theorie wurde ein Parallelismus zwischen der Harnsäureausscheidung und der Zahl der Leukocyten im Blute angenommen und nachzuweisen gesucht.

Diese Leukocytosetheorie leitete ziemlich alle Untersuchungen auf diesem Gebiete durch lange Zeit. Ich habe zwar gegen dieselbe gleich nach ihrer Veröffentlichung Einwände erhoben ${ }^{2}$ ), deren tatsächliche Gültigkeit aber erst viel später von anderen erkannt und allmählich auch erwiesen wurde. Gegenwärtig ist die Unhaltbarkeit jener Leukocytosetheorie "von verschiedenen Seiten vollständig dargetan, ohne dass gleichwohl eine andere wahrscheinlichere an ihre Stelle getreten wäre" [Sivén" $\left.\left.{ }^{3}\right)\right]$. Endlich ist die Leukocytosetheorie von ihrem Urheber selbst als "nicht bewährt" aufgegeben worden ${ }^{4}$ ).

Bei dieser Sachlage finde ich mich veranlasst, aus meiner durch 18 Jahre in dieser Frage beobachteten Zurückhaltung hervorzutreten und meine ursprüngliche T'heorie der Harnsäurebildung beim Menschen von neuem vorzutragen; und dies um so mehr, als sich in der neueren Literatur bestimmte Anklänge an dieselbe vernehmen lassen, und als es mir jetzt, bei dem gegenwärtigen Stande der Untersuchungen in dieser Frage, möglich ist, die Einwände, welche gegen diese Theorie erhoben worden sind, kritisch zu beleuchten und womögtich zu zerstreuen. Ich kann auch jetzt aus meiner Reserve hervortreten, da es nicht mehr um die Leukocytosetheorie zu tun ist, und ich vollends das tatsächliche Verdienst meines verehrten Kollegen Horbaczewski

1) Horbaczewski, Sitzungsber. d. kaiserl. Akad. Wien Bd. 98, Juli 1889. Bd. 100, April 1891.

2) F. Marě̌, Zur Theorie der Harnsäurebildung im Säugetierorganismus. Sitzungsber. d. kaiserl. Akad. d. Wissensch. in Wien Bd. 101. Januar 1892.

3) O. Sivén, Skand. Arch. f. Physiol. Bd. 18 S. 181. 1906.

4) J. Horbaczewski, Chemie lékarská Bd. 3 S. 536. Praba 1908. 
in der Frage des Zusammenhanges der Harnsäure mit den Xantlinkörpern und den Nukleinen hervorheben kann. Für mich selbst aber nelme ich in Anspruch, früher den physiologischen Nachweis erbracht zu haben, dass die Harnsäure ein Produkt der Körperzellen ist, unabhängig vom Eiweissgehalt der Nahrung. Der von mir aufgestellte Satz:

„Die Harnsäure ist ein Produkt des Stoffwechsels in den lebenden Zellen, wobei namentlich die Nukleine der Zellkerne beteiligt sind" kann als gemeinsame Frucht zweier verschiedener, wörtlich unter einem Dache geführter Arbeitsrichtungen betrachtet werden.

Die erwähnten Anklänge an meine Untersuchungen über den Ursprung der Harnsäure beim Menschen ertönten zunächst von seiten einiger Autoren, welchen die Grundlage der Leukocytosetheorie, das ist der angenommene Parallelismus zwisehen der Harnsäuremenge und der Zahl der Leukocyten im Blute, zweifelhaft erschien. So haben Milroy und Malcolm ${ }^{1}$ ) meine Stellungnahme gegen die Leukocytosetheorie beachtet. $\mathrm{Hopkins}$ und $\mathrm{Hope}^{2}$ ) haben dann meine Einwände gegen die Leukocytosetheorie näher besprochen und wiederholten und bestätigten einen Teil meiner Untersuchungen, ohne aber den von mir angegebenen Zusammenhang der Harnsäurevermehrung nach Fleischeinnahme mit der durch diese angeregten Tätigkeit der Verdauungsdrüsen anzunehmen, aus Gründen, die ich noch zu erwähnen und zu erwägen haben werde. Aus denselben Gründen haben auch Sivén ${ }^{3}$ ) und Burian mit $S c h u r^{4}$ ) sich gegen meine Theorie ablehnend verhalten, obgleich sie meinen Untersuchungen selbst eine besondere Aufmerksamkeit gewidmet haben. Zuletzt hat $\mathrm{W}$ iener $\mathrm{r}^{5}$ ) meine Untersuchungen und besonders meine Theorie einer eingehenden Würdigung unterzogen und diese Theorie in einer ganz allgemeinen Fassung auch angenommen, dass nämlich die Harnsäure ein Produkt des physiologischen Stoffwechsels der Zellen ist, an welchem die Zellkerne beteiligt sind. Ob dabei spezielle

1) Milroy and Malcolm, The Journ. of Physiol. vol. 23 p. 221. 1898.

2) Hopkins and Hope, The Journ. of Physiol. vol. 23 p. 271. 1898.

3) Sivén, Skand. Arch. f. Physiol. Bd. 11 S. 143. 1901.

4) Burian und Schur, Pfläger's Arch. f. d. ges. Physiol. Bd. 80 S. 251.1900.

5) H. Wiener, Ergebnisse der Physiologie von A sher und Spiro Bd. 1 H. 1 S. 582.1902. 
Org̣ane, wie die Verdauungsdrüsen, eine grössere Rolle spielen, das liess Wiener vorläufig dahingestellt. Aber der Zusammenhang der Harnsäurebildung mit der Tätigkeit der Verdauungsdrüsen war gerade der tatsächliche Grund, auf welchem ich jene 'Theorie aufgestellt habe; wird dieser Grund hinfällig, so sinkt die Theorie zu einer blossen ganz allgemeinen Annahme herab.

In dieser ganz allgemeinen Fassung wird diese Theorie von vielen auch angenommen, als wenn sie keinen besonderen tatsächlichen Grund hätte, ausgenommen vielleicht die nicht bezweifelte individuelle Konstanz der Harnsäure bei verschiedenen Individuen. So sagt z. B, Abderhalden ${ }^{1}$ ): „Im endogenen Harnsäurewert haben wir einen direkten Ausiruck für den Umfang und die Grösse der Zellarbeit, die, wie wir aus mancherlei Beobachtungen wissen, für jedes Einzelwesen eine ganz besonders geregelte und eingestellte ist." Und $O$. Weiss ${ }^{2}$ ) führt einfach an: "Vielmehr hat sich die Mehrzahl der Forscher der Anschauung angeschlossen, die einmal von Mareš ausgesprochen ist, dass nämlich die Harnsäure das Endprodukt des Stoffwechsels der Zellkerne ist."

So scheint diese Anschaung grundlos in der Luft zu schweben, wie eine Sage, weil ihr tatsächlicher Grund verkannt oder gar ganz unbekannt ist. Es ist also nicht zu verwundern, wenn einige dasjenige ganz ignorieren, was andere als annehmbar anerkennen. Hammarsten ${ }^{3}$ ) z. B. führt meine Untersuchungen unter den Belegen dafür an, dass bei vegetabilischer Kost die Harnsäuremenge kleiner ist als bei Fleischnahrung, wovon aber darin überhaupt keine Rede ist. Andere wieder halten meine Untersuchungen für „nicht ganz einwandfrei" und glauben "von diesen unvollkommenen Versuchen " absehen zu können ${ }^{4}$ ). Einige tun dies wenigstens stillschweigend ${ }^{5}$ ).

Als verantwortlicher Urheber jener "von der Mehrzahl der Forscher angenommenen Anschauung" fühle ich mich verpflichtet, den tatsächlichen Grund dieser Anschauung von neuem darzulegen, sobald Zweifel um denselben laut werden. Ich kann dies jetzt um so mehr,

1) Abderhaldeu, Lehrb. d. physiol, Chemie, 2. Aufl, S. 390.1909.

2) O. Weiss, Nagel's Handb. d. Pbysiol. Bd. 2. (1) S. 354.1906.

3) Hammarste n, Lehrb. d. physiol. Chemie, 7. Aufl., S. 664. 1910.

4) $\mathrm{Ka} \mathrm{ufmann}$ und Mohr, Deutsches Arch. f. klin. Medizin Bd. 74 S. 141. 1902.

5) Magnus Levy, v. Noorden's Handb. d. Pathol. d. Stoffwechsels, 2. Aufl., 1907. - A. Ellinger, Biochemie von Oppenheimer Bd. 3 (1) S. 574: 
als ich in der Lage bin, den stïrksten Einwand, der gegen den von mir behaupteten Zusammenhang der Harnsäurevermehrung mit der durch Fleicheinnahme angeregten Verdaungsslrüsentätigkeit erhoben wurde, zerstreuen zu können, und zwar durch eine kritische Zusammenstellung meiner ursprünglichen Versuche mit späteren Untersuchungen anderer Autoren, sowie durch eigene neue Versuche, die ich in dieser Richtung veranlasst habe, und deren Ergebnis in einem folgenden Artikel dargelegt werden wirl.

I. Individuelle Konstanz der Harnsäuremenge bei sehr verschiedener Menge des ausgeschiedenen Gesamtstickstoffs im nüchternen Zustande.

Um die Verhältnisse zwischen der Harnsäuremenge und dem ausgesehiedenen Harnstickstoff zu verfolgen, machte ieh 22 Versuche an fünf Männern im Alter von 13-45 Jahren, und zwar im nüchternen Zustande. Die Versuche begannen 12 Stunden nach der letzten Nahrungsaufnahme und dauerten bis zur 24., einige Male bis zur 27. Stunde. Der Harn wurde in dreistündigen Perioden gesammelt und analysiert. Die Harnsäure wurde nach SalkowskiLudwig, der Gesamtstickstoff nach Kjeldahl bestimmt. Es war wohl eine der ersten Untersuchnngen des Harns mittelst dieser bis jetzt unübertroffenen Methorlen. Die Ergebnisse sind in der folgenden Tabelle I (S. 65) dargestellt.

Diese Versuche zeigen, dass im nüchternen Zustande die von einer Versuchsperson ausgeschiedene Harnstickstoffmenge an verschiedenen Tagen in sehr weiten Grenzen bis nahezu um $100 \%$ variieren kann, dass dagegen die zu gleicher Zeit ausgeschiedene Harnsäuremenge nur wenig um einen Mittelwert herumschwankt, welcher bei verschiedenen Versuchspersonen verschieden ist und individuell konstant erscheint. So variert bei der Versuchsperson A die Harnstickstoffausscheidung in 15 Stunden nüchternen Zustandes an verschiedenen Tagen zwischen 6,078-10,005 g, die Harnsäuremenge aber erscheint zu gleicher Zeit, an ziemlich weit entfernten Tagen, ganz gleichmässig, indem sie sich sehr nahe am Durchschnittswerte von $0,2659 \mathrm{~g}$ hält, woraus sich für diese Versuchsperson ein individuell konstanter Wert von $17,7 \mathrm{mg}$ Harnsäure in einer Stunde ergeben würde. Bei der Versuchsperson B variiert die Harnstickstoffausseheidung zwischen $5,15-9,47 \mathrm{~g}$ in 15 Stunden nüchternen Zustandes, der Harnsäurewert aber hält sich knapp um 
Der physiologische Protoplasmastoffwechsel und die Purinbildung.

65

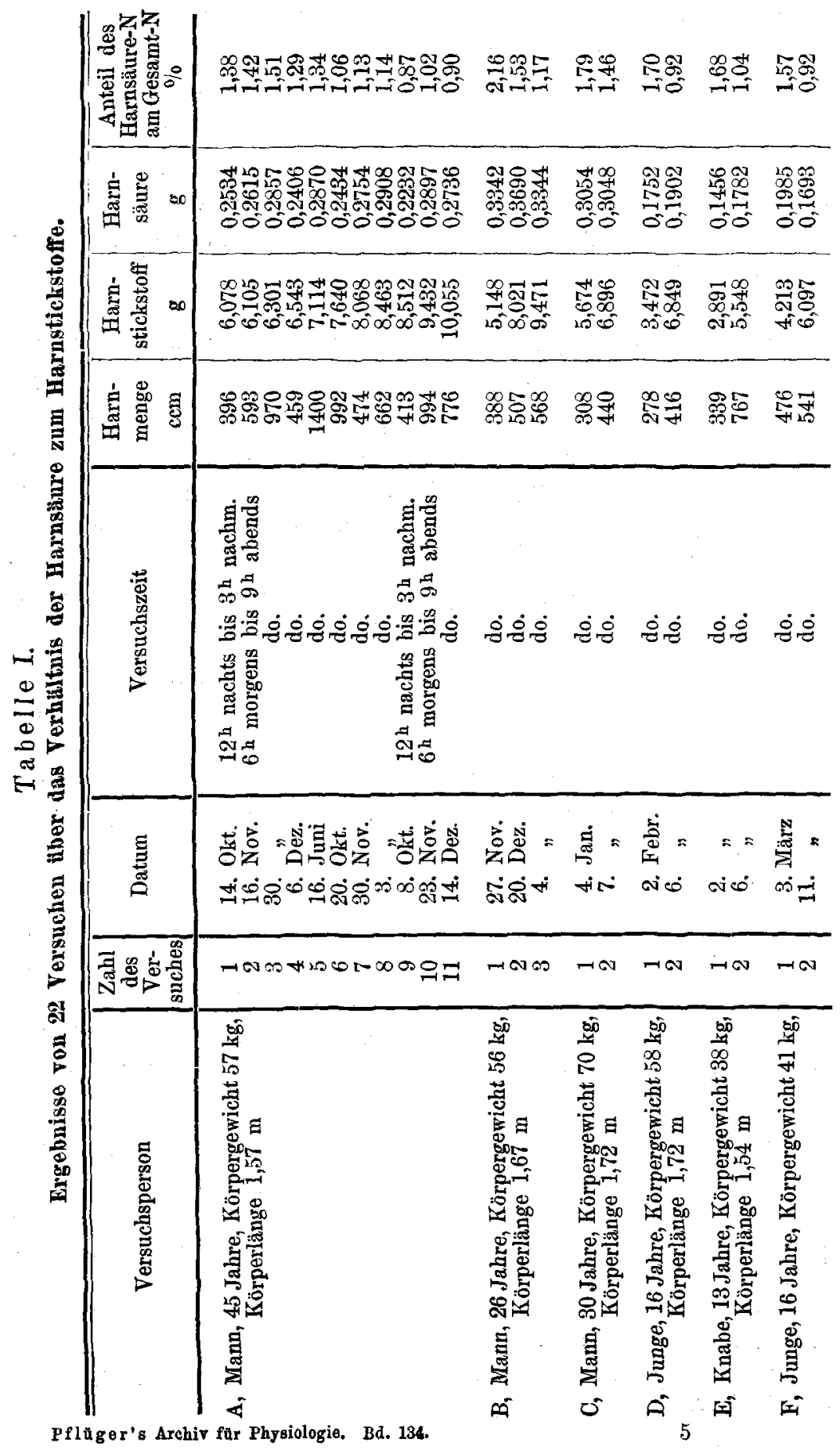


$0,3458 \mathrm{~g}$, oder $23 \mathbf{m g}$ in 1 Stunde. Bei der Versuchsperson $\mathbf{C}$ Harnstickstoff $5,7-6,9-\mathrm{g}$, Harnsäure $0,305 \mathrm{~g}$ in 12 Stunden oder 25,4 $\mathrm{mg}$ in 1 Stunde. Bei der Versuchsperson D Harnstickstoff $3,5-6,3 \mathrm{~g}$, Harnsäure $0,1827 \mathrm{~g}$ in 12 Stunden, $15,2 \mathrm{mg}$ in 1 Stunde. Bei der Versuchsperson E Harnstickstoff $2,9-5,5 \mathrm{~g}$, Harnsäure $0,1619 \mathrm{~g}$ in 12 Stunden, $13,5 \mathrm{mg}$ in 1 Stunde. Bei der Versuchsperson $F$ Harnstickstoff $4,2-6,1 \mathrm{~g}$, Harnsäure $0,1839 \mathrm{~g}$ in 12 Stunden, $15,3 \mathrm{mg}$ in 1 Stunde.

Die grossen Variationen in der Harnstickstoffausscheidung wurden absichtlich dadurch hervorgerufen, dass die Versuchsperson mit der letzten Mahlzeit, 12 Stundep vor Beginn jedes Versuches, das einemal sehr wenig, das anderemal sehr viel Eiweiss (Fleisch) zu' sich genommen hatte. Der Einfluss dieser eiweissreichen Nabrung zeigte sich von der 12.--24. Stunde nach deren Einnahme noch sehr deutlich in der Harnstickstoffmenge (Harnstoff); auf die Harnsäuremenge aber war zu dieser Zeitperiode kein Einfluss der Nahrung mehr zu beobachten. Danach erscheint die Harnsäuremenge von der vorher eingenommenen Eiweiss-(Fleisch-) menge ganz unabhängig zu einer Zeitperiode, wo der Finfluss dieser Eiweissmenge auf die Menge des Harnstickstoffes (Harnstoffes) noch sehr deutlich ist.

Der supponierte Parallelismus zwischen Harnstoffmenge, Harnsäuremenge und der Menge des Nahrungseiweisses besteht also nicht. Jene beiden stickstoffhaltigen Stoffwechselprodukte haben offenbar verschiedenen Ursprung. Der $\mathrm{Harnst}$ of $\mathrm{f}$ entspringt wabrscheinlich aus dem verdauten und resorbierten Nahrungseiweiss, da seine Menge mit diesem steigt und sinkt. Aber nicht die Harnsäure diese entspringt aus einer individuell charakterisierten Quelle.

Diese Tatsachen wurden später von anderen Untersuchern bestätigt. Salkowski ${ }^{1}$ ) (mit Spilker) fand bei verschiedener Ernährung die Harnstoffmenge im höchsten Grade von der Grösse der Eiweisszufuhr abhängig, während die absolute Quantität der Harnsäure von der Nahrung unabhängig und ohne Zweifel individuell sich zeigte. Burian und $\mathrm{Schur}{ }^{2}$ ) traten besonders für die individuelle Konstanz des endogenen Harnpurinwertes ein, den sie für ein und

1) Salkowski, Virchow's Arch. Bd. 117 S. 570. 1889. -- Zentralbl. f. d. med. Wissensch. 1890 S. 360 (Autoref.).

2) Burian und Schur, Pflüger's Arch. f. d. ges. Physiol. Bd. 80 S. 292. 1900 . 
dasselbe Individuum als eine physiologische Konstante bezeichneten. Diesen Wert wollten sie aber nicht im Hungerzustande bestimmt haben, wie ich es tat, sondern bei zureichender purinfreier Ernährung, aus Gründen, auf welche ich zurückkommen werde.

Obgleich die in einer 12-15 stündigen Periode des nüchternen $\mathrm{Zu}$ ustand es ausgeschiedene Gesamtmenge der Harnsäure einen für jede Versuchsperson konstanten Wert aufweist, so ist doch die in den einzelnen dreistündigen Phasen dieser Periode ausgeschiedene Harnsäuremenge nicht gleichmässig, welches von mir zunächst nicht berücksichtigte Verhältnis von Si ve en ${ }^{1}$ ) her vorgehoben wurde, welcher dann auch auf die Verminderung der Harnsäureausscheidung während der Nacht und auf deren Vergrösserung während des Vormittags hingewiesen hat. Diese Verbältnisse treten auch deutlich in meinen Versuchen hervor, wie die Versuche A 1 und 9 , welche úm Mitternacht begannen, im Vergleiche mit den Versuchen 2 und 8 , welche um 6 Uhr morgens begannen und entsprechende Gesamtstickstoffmengen aufweisen, zeigen können.

(Siehe Tabelle II auf S. 68.)

Aus diesen Beispielen ist zu ersehen, dass die Harnsäureausscheidung während der Nachtstunden geringer ist als in den Vormittagsstunden (A 1,9), und dass dieselbe gegen abend wieder geringer wird (A 2, 8). So erklärt es sich, dass gerade die zwei um Mitternacht beginnenden Versuche kleinere Harnsäurewerte aufweisen als die Tagesversuche. Tatsächlich zeigen auch die reinen Tagesversuche B-F geringere Schwankungen im Harnsäturegesamtwerte als die gemischten Versuche A.

Es entstand nun für mich die Frage, mit welchen Umständen die Grösse des individuellen Harnsäurewertes zusammenhängen könnte. Ich dachte zunächst an das Alter der Versuchspersonen. Berechnet man den individuellen Harnsäurewert jeder Versuchsperson für $1 \mathrm{~kg}$ Körpergewicht und 24 Stunden und ordnet diese Werte nach dem Alter der Versuchspersonen, so bekommt die auf S. 68 Tab. III befindliche Übersicht.

Sehen wir vorläufig von der Versuchsperson D ab, so könnten wir ein Steigen des individuellen Harnsäurewertes bis zum 26. Lebensjahre und dann mit steigendem Lebensalter ein Herabsinken desselben annehmen und dieses Verhältnis mit der Körperentwicklung

1) V. O. Sivén, Skand. Arch. f. Physiol. Bd. 11 S. 143.1901. 


\section{Tabelle II.}

Verlauf der Harnsäureausscheidung in dreistündigen Phasen der Versuche $A 1,2,0,8$.

\begin{tabular}{|c|c|c|c|c|c|c|}
\hline \multirow[b]{2}{*}{ Stundenphase } & \multicolumn{3}{|c|}{ A 1} & \multicolumn{3}{|c|}{ A 2} \\
\hline & $\begin{array}{c}\text { Harn- } \\
\text { menge } \\
\text { ccm }\end{array}$ & $\begin{array}{c}\text { Harn- } \\
\text { stickstoff } \\
g\end{array}$ & $\begin{array}{l}\text { Harn- } \\
\text { säure } \\
\mathrm{g}\end{array}$ & $\begin{array}{c}\text { Harn- } \\
\text { menge } \\
\text { ccm }\end{array}$ & $\begin{array}{c}\text { Harn- } \\
\text { stickstoff } \\
\mathrm{g}\end{array}$ & $\begin{array}{c}\text { Harn- } \\
\text { säure } \\
\mathbf{g}\end{array}$ \\
\hline $\begin{array}{lrr}\text { Nachts } & 12-3 & \mathrm{~h} \\
& 3-6 & \mathrm{~h} \\
\text { Morgens } & 6-9 & \mathrm{~h} \\
\text { Vorm. } & 9-12 \mathrm{~h} \\
\text { Mittags } & 12-3 & \mathrm{~h} \\
\text { Nachm. } & 3-6 & \mathrm{~h} \\
\text { A bends } & 6-9 & \mathrm{~h} \\
\end{array}$ & $\begin{array}{l}88 \\
84 \\
96 \\
70 \\
58 \\
- \\
-\end{array}$ & $\begin{array}{l}1,382 \\
1,331 \\
1,315 \\
1,102 \\
0,957 \\
- \\
- \\
\end{array}$ & $\begin{array}{c}0,0422 \\
0,0478 \\
0,0622 \\
0,0510 \\
0,0502 \\
= \\
= \\
\end{array}$ & $\begin{array}{l}\overline{-} \\
\overline{210} \\
147 \\
100 \\
74 \\
62 \\
\end{array}$ & $\begin{array}{l}\overline{-} \\
1, \overline{712} \\
1,377 \\
1,176 \\
0,900 \\
0,940 \\
\end{array}$ & $\begin{array}{r}- \\
0, \overline{0} 85 \\
0,0587 \\
0,0527 \\
0,0252 \\
0,0464\end{array}$ \\
\hline Summa: & 396 & 6,078 & 0,2534 & 593 & 6,105 & 0,2615 \\
\hline
\end{tabular}

\begin{tabular}{|c|c|c|c|c|c|c|}
\hline \multirow[b]{2}{*}{ Stundenphase } & \multicolumn{3}{|c|}{ A 9} & \multicolumn{3}{|c|}{ A 8} \\
\hline & $\begin{array}{c}\text { Harn- } \\
\text { menge } \\
\text { ccm }\end{array}$ & $\begin{array}{c}\text { Harn- } \\
\text { stickstoff } \\
\mathbf{g}\end{array}$ & $\begin{array}{l}\text { Harn- } \\
\text { säure } \\
\mathbf{g}\end{array}$ & $\begin{array}{l}\text { Harn- } \\
\text { menge } \\
\text { ccm }\end{array}$ & $\begin{array}{c}\text { Harn- } \\
\text { stickstoff } \\
\mathrm{g}\end{array}$ & $\begin{array}{c}\text { Harn- } \\
\text { säure } \\
\mathbf{g}\end{array}$ \\
\hline \begin{tabular}{lrr} 
Nachts & $12-3$ & $\mathrm{~h}$ \\
\multicolumn{1}{c}{} & $3-6$ & $\mathrm{~h}$ \\
Morgens & $6-9$ & $\mathrm{~h}$ \\
Vorm. & $9-12 \mathrm{~h}$ \\
Mittags & $12-3$ & $\mathrm{~h}$ \\
Nachm. & $3-6$ & $\mathrm{~h}$ \\
Abends & $6-9$ & $\mathrm{~h}$ \\
\end{tabular} & $\begin{array}{r}100 \\
76 \\
85 \\
80 \\
72 \\
- \\
-\end{array}$ & $\begin{array}{l}1,654 \\
1,983 \\
1,725 \\
1,577 \\
1,573 \\
-\end{array}$ & $\begin{array}{l}0,0375 \\
0,0457 \\
0,0481 \\
0,0509 \\
0,0410 \\
- \\
-\end{array}$ & $\begin{array}{l}- \\
230 \\
150 \\
106 \\
102 \\
74\end{array}$ & $\begin{array}{l}- \\
2, \overline{436} \\
1,768 \\
1,519 \\
1,466 \\
1,274\end{array}$ & $\begin{array}{c}\overline{-} \\
0, \overline{0} 51 \\
0,0507 \\
0,0604 \\
0,0501 \\
0,0445\end{array}$ \\
\hline Summa: & 413 & 8,512 & 0,2232 & 662 & 8,463 & 0,2908 \\
\hline
\end{tabular}

\section{Tabelle III.}

Vergleichung der individuellen Harnsäurewerte nach dem Alter und dem Körpergewicht der Versuchspersonen.

\begin{tabular}{c|c|c|c}
\hline \multirow{2}{*}{ Versuchsperson } & Alter & Körpergewicht & $\begin{array}{c}\text { Harnsäurewert für } \\
1 \mathrm{~kg} 24 \text { Std. } \\
\mathrm{mg}\end{array}$ \\
\hline \multirow{2}{*}{ Jahre } & 13 & 38 & 8,53 \\
F & 16 & 41 & 8,96 \\
D & 16 & 58 & 6,29 \\
B & 26 & 56 & 9,86 \\
C & 30 & 70 & 8,78 \\
A & 45 & 57 & 7,40
\end{tabular}


in Zusammenhang bringen. Zur Behduptung eines solchen Verhältnisses aber genügen diese Versuche nicht; es müssten solcbe Versuche an einer und derselben Versuchsperson im verschiedenen Alter angestellt werden, was nicht leicht zu verwirklichen ist. Bei yerschiedenen Versuchspersonen kann jenes Verhältnis durch ungleichmässige Entwicklung verdeckt werden, wie es bei der Versuchsperson $\mathrm{D}$ der Fall ist. $\mathrm{D}$ und $\mathrm{F}$ stehen beide im 16 . Lebensjahre; während aber $\mathbf{F}$ seinem Alter entsprechend entwickelt ist, hat D eine im Verhältnis zum Körpergewicht übermässige Körperlänge $(58 \mathrm{~kg}-1,72 \mathrm{~m})$, das ist ein Übergewicht des Skeletts über die anderen Organe. Besteht nun wirklich ein Zusammenhang zwischen Harnsäurebildung und Tätigkeit der protoplasmatischen Organe, so würde daraus der überaus niedrige Harnsäurewert bei dieser Versuchsperson erklärlich sein.

Der individuell konstante Harnsäurewert könnte im allgemeinen mit der individuell verschiedenen und mit dem Alter sich verändernden Aktivität des Protoplasmas überhaupt im Zusammenhange sein. Am intensivsten ist diese Aktivităt bei der Bildung und dem Wachstum des Körpers während der embryonalen Entwicklung. Bei Neugeborenen ist tatsächlich die Harnsäureausscheidung sehr gross. Ich habe darüber nähere Untersuchungen angestellt an Harnen von Neugeborenen, die mir aus der Gebäranstalt in Prag zugestellt wurden, und zwar gleich in den ersten Lebensstunden bis etwa zum 14. Lebenstage; leider waren die zugehörigen Zeitperioden nicht genau zu bestimmen, ausser in einem Falle, so dass die Bestimmungen auf das Verbältnis der Harnsäure zum Gesamtstickstoff des Harns beschränkt sind. Die Ergebnisse befinden sich in der Tab. IV auf S. 70.

Die Harnsäureproduktion ist also beim Neugeborenen ungemein gross; in den ersten Lebensstunden werden mehr als $8 \%$ des gesamten Harnstickstoffes in Form von Harnsäure ausgeschieden, während dieses Verhältnis beim Erwachsenen kaum $2 \%$ erreichen dürfte. In den folgenden Lebenstagen sinkt dieses Verbältnis, indem die Harnsäuremenge sich vermindert. Am achten Lebenstage beträgt jenes. Verhältnis etwa $5 \%$, am 14. Tage etwa $3 \%$. Im Versuche $a$ erscheint der gleich nach der Geburt in den ersten 12 Stunden gelassene Harn ziemlich arm an Stickstoff und Harnsäure; erst in den darauffolgenden 12 Stunden zeigt er entsprechende Mengen davon. Im Versuche d wurde die 24 stündige Harnmenge möglichst genau bestimmt, so dass hier die absoluten Mengen des gesamten Harn- 
Tabelle IV.

Das Verhältnis zwìschen Harnstickstoff nnd Harnsäureausscheidung beim Nêugeborenen.

\begin{tabular}{|c|c|c|c|c|c|}
\hline Nationale & $\begin{array}{l}\text { Lebens- } \\
\text { periode }\end{array}$ & $\begin{array}{l}\text { Harn- } \\
\text { menge } \\
\text { ccm }\end{array}$ & $\begin{array}{l}\text { Gesamt- } \\
\text { stickstoff } \\
\quad \because g\end{array}$ & $\begin{array}{l}\text { Harn- } \\
\text { säure } \\
\mathrm{g}\end{array}$ & $\begin{array}{l}\text { Anteil des } \\
\text { Harnsäure-N } \\
\text { am Gesamt-N } \\
\% \%\end{array}$ \\
\hline a) geb. 17, Dez., Gew. $3100 \mathrm{~g}$ & $\underset{12-24}{0-12 \text { Std }}$ & $\begin{array}{l}20 \\
16\end{array}$ & $\begin{array}{c}0,0503 \\
0,1728\end{array}$ & $\begin{array}{l}0,0082 \\
0,0392\end{array}$ & $\begin{array}{l}5,49 \\
7,56\end{array}$ \\
\hline b) geb. 19. Dez, Gew, $2770 \mathrm{~g}$ & $0-24 \quad$ " & 20 & 0,180 & 0,0439 & 8,13 \\
\hline c) geb. 8. Dez., Gew. $3850 \mathrm{~g}$ & $\begin{array}{c}12-30 \\
4 . \mathrm{Ta} \\
5 . \\
6 . " \\
7 . " \\
8 . "\end{array}$ & $\begin{array}{r}- \\
90 \\
192 \\
220 \\
210 \\
136\end{array}$ & $\begin{array}{l}0,2408 \\
0,189 \\
0,238 \\
0,245 \\
0,253 \\
0,261\end{array}$ & $\begin{array}{l}0,0612 \\
0,0275 \\
0,0365 \\
0,0224 \\
0,0277 \\
0,0312\end{array}$ & $\begin{array}{l}8,46 \\
4,85 \\
5,11 \\
3,05 \\
3,64 \\
3,98\end{array}$ \\
\hline $\begin{array}{l}\text { d) geb. 19. Jan., Gew. } 4150 \mathrm{~g} \\
\text { e) } \\
\text { f) }\} \text { milling }\left\{\begin{array}{l}\text { Gew. } 2860 \mathrm{~g} \\
\text { Gew. } 2830 \mathrm{~g}\end{array}\right.\end{array}$ & $\begin{array}{r}\text { 8. " } \\
\text { 14. " } \\
14 .\end{array}$ & 200 & $\begin{array}{l}0,338 \\
0,185 \\
0,161\end{array}$ & $\begin{array}{l}0,049 \\
0,0174 \\
0,0126\end{array}$ & $\begin{array}{l}4,9 \\
3,13 \\
2,56\end{array}$ \\
\hline
\end{tabular}

stickstoffes und der Harnsäure zu ersehen sind. Hiernach entfielen bei diesem 8 tägigen Säugling auf $1 \mathrm{~kg}$ Körpergewicht $11,8 \mathrm{mg}$.Harnsäure und $81,4 \mathrm{mg}$ Gesamtharnstickstoff in 24 Stunden, während der höchste beim Erwachsenen (B) gefundene Harnsäurewert nur $\mathbf{9 , 8 6} \mathbf{~ m g}$ betrug, dagegen aber der geringste Gesamtstickstoffwert bei demselben $147 \mathrm{mg}$ ausmachte. Beim Säugling erscheint also die Gesamtstickstoffausscheidung bedeutend geringer, die Harnsăureausscheidung bedeutend grösser als beim Erwachsenen. Der: Säugling vergeudet seinen Nahrungsstickstoff nicht in so hohem Grade whe der Erwachsene.

\section{Einfluss der Eiweisseinnahme auf die Ansscheidung des Harnstickstoffes und der Harnsäure.}

Nach älteren übereinstimmenden Beobachtungen bewirkt Einnahme von eiweissreicher Nahrung Vermehrung der Harnsäureausscheidung ebenso wie des Harnstoffes; was eben zu der Annahme geführt hat, dass beide Stoffe vom Nahrungseiweiss herrühren. Das Ergebnis meiner eben mitgeteilten Untersuchung stellte mich vor die Aufgabe, dieses Verhältnis aufzuklären. $\mathrm{Zu}$ diesem Zwecke liess ich die Versuchsperson zh einer bestimmten Stunde des nüchternen Zustandes viel Fleisch verzehren; indem ich den Verlauf der Harnsäure- sowie der Gesamtstickstoffausscheidung von Stunde zu Stunde bestimmte. Das Ergebnis war wie folgt: 
Tabelle V.

Einfluss der Fleischeinnahme auf die Ausscheidung des Gesantstickstoffes und der Harnsäure.

\begin{tabular}{|c|c|c|c|c|c|c|}
\hline Anmerkung & $\begin{array}{c}\text { Zabl } \\
\text { des } \\
\text { Ver- } \\
\text { suches }\end{array}$ & Stundenphase & $\begin{array}{l}\text { Harn- } \\
\text { menge } \\
\text { ccm }\end{array}$ & $\begin{array}{l}\text { Gesamt- } \\
\text { stickstoff } \\
\mathrm{g}\end{array}$ & $\begin{array}{l}\text { Harn- } \\
\text { sâure } \\
\mathrm{g}\end{array}$ & $\begin{array}{c}\text { Anteil des } \\
\text { Harnsäure-N } \\
\text { am Gesamt-N } \\
0 \%\end{array}$ \\
\hline \multirow{14}{*}{$\begin{array}{l}\text { Versuchspers. A } \\
\text { Fleischeinnahme }\end{array}$} & 1 & $6-7 \mathrm{~h}$ morg & 53 & 0,534 & $0,02 \theta 3$ & 1,45 \\
\hline & $\begin{array}{l}2 \\
3\end{array}$ & $\begin{array}{l}7-8 \mathrm{~h} \\
8-9 \mathrm{~h}\end{array}$ & $\begin{array}{l}62 \\
50\end{array}$ & $\begin{array}{l}0,559 \\
0,462\end{array}$ & $\begin{array}{l}0,0219 \\
0,0190\end{array}$ & $\begin{array}{l}1,30 \\
1,38\end{array}$ \\
\hline & 4 & $9-10 \mathrm{~h}$ & 35 & 0,421 & 0,0240 & 1,90 \\
\hline & 5 & $10-11 \mathrm{~h}$ & 36 & 0,546 & 0,0465 & 259 \\
\hline & 6 & $11-12 \mathrm{~h}$ & 50 & 0,760 & 0,0739 & $\mathbf{3}, 24$ \\
\hline & 7 & $12-1 \mathrm{~h}$ & 58 & 0,933 & 0,0739 & 2,64 \\
\hline & 8 & $1-2 \mathrm{~h}$ & 94 & 1,261 & 0,0831 & 2,19 \\
\hline & 9 & $2-3 h$ & 120 & 1,394 & 0,0730 & 1,74 \\
\hline & 10 & $3-4 \mathrm{~h}$ & 136 & 1,479 & 0,0700 & 1,58 \\
\hline & 11 & $4-5 k$ & 146 & 1,494 & 0,0675 & 1,50 \\
\hline & 12 & $5-6 \mathrm{~h}$ & 120 & 1,433 & 0,0598 & 1,38 \\
\hline & 13 & $6-7 \mathrm{~h}$ & 100 & 1,305 & 0,0454 & 1,15 \\
\hline & 14 & $7-8 \mathrm{~h}$ & 86 & 1,132 & 0,0346 & 1,01 \\
\hline & 15 & $8-9 h$ & 55 & 0,944 & 0,0240 & 0,85 \\
\hline \multirow{11}{*}{$\begin{array}{l}\text { Versuchspers. } \mathbf{F} \\
\text { Fleischeinnahme }\end{array}$} & 1 & & & 0,944 & 0,0240 & 0,85 \\
\hline & $\begin{array}{l}2 \\
3\end{array}$ & $6-9 \mathrm{~h}$ & 453 & $\begin{array}{l}0,359 \\
0,359\end{array}$ & $\begin{array}{l}0,0110 \\
0,0110\end{array}$ & $\begin{array}{l}1,02 \\
1,02\end{array}$ \\
\hline & 4 & $9-10 h$ & 61 & 0.273 & 0,0137 & 1.67 \\
\hline & $\begin{array}{l}4 \\
5\end{array}$ & $10-11 \mathrm{~h}$ & 45 & 0,391 & 0,0222 & 2,23 \\
\hline & 6 & $11-12 \mathrm{~h}$ & 51 & 0,476 & 0,0333 & $\mathbf{2}, \mathbf{3 3}$ \\
\hline & 7 & $12-1$ h & 48 & 0,502 & 0,0348 & $\mathbf{2 , 3 0}$ \\
\hline & 8 & $1-2 h$ & 68 & 0,764 & 0,0456 & 1,99 \\
\hline & 9 & $2-3 \mathrm{~h}$ & 51 & 0,675 & 0,0312 & 1,54 \\
\hline & 10 & $3-4 h$ & 55 & 0,730 & 0,0303 & 1,38 \\
\hline & 11 & $4-5 h$ & 56 & 0,817 & 0,0280 & 1,14 \\
\hline & 12 & $5-6 \mathrm{~h}$ & 52 & 0,764 & 6,0233 & 1,01 \\
\hline
\end{tabular}

Es zeigte sich also, dass Einnahme von Fleisch wohl eine Vermehrung der Harnsäureausscheidung bewirkt, dass dieselbe aber mit der Vermehrung des Gesamtstickstoffes nicht parallel verläuft, sondern dieser etwa um 3 Stunden vorangeht. Die Vermehrung der Harnsäureausscheidung beginnt gleich in der ersten Stunde nach der Fleicheinnahme und erreicht in der fünften Stunde etwa ihr Maximum, um dann allmählich abzusinken. Die Vermehrung des Gesamtstickstoffes beginnt etwa in der dritten Stunde nach der Fleischeinnahme deutlich zu werden und erreicht ihr Maximum erst in der áchten Stunde, um sich dann ziemlich lange auf der Höhe zu erhalten. So erklärt es sich, dass im nüchternen Zustande der Harnsâurewert anf das individuell konstante Maass bcreits zurückgekommen 
ist, wo die Gesamtstickstoffmenge noch unter dem Einflusse der Eiweissmenge der vor dem Beginne des Versuches eingenommenen Nahrung steht.

Das zeitliche Verhältnìs der Harnsäure- und der Gesamtstickstoffausscheidung nach Fleischeinnabme führte mich auf den Gedanken, dass die Harnsäurevermehrung mit der Tätigkeit der durch die Fleicheinnahme angeregten Verdauungsdrüsen zusammenhängen könnte, während die Vermehrung des Gesamtstickstoffes (Harnstoffes) von dem verdauten und absorbierten Nahrungseiweiss herrühren würde. Diese Frage war durch die angeführten Versuche selbst nicht $\mathrm{zu}$ entscheiden, weil doch immer die Möglichkeit bestand, dass die Harnsäurevermehrung von dem eingenommenen Fleische selbst herrühren könnte, welcher Einwand später tatsächlich erhoben worden ist. Gleichsam in Ahnung dieses Einwandes fühlte ich die Notwendigkeit, die Verdauungsdrüsen durch einen nicht stofflichen, inadäquaten Reiz zur Tätigkeit anzuregen, um zu sehen, ob eine solche sozusagen reine Drüsentätigkeit mit Harnsäurevermehrung verbunden ist oder nicht. Zu diesem Zwecke wählte ich das Pilokarpin. Ich gab also der Versuchsperson zu einer bestimmten Stunde des nüchternen Zustandes anstatt des Fleiches eine subkutane Injektion von $0,012-0,02 \mathrm{~g}$ Pilokarpin und verfolgte ebenso die Gesamtstickstoff- und Harnsäureausscheidung von Stunde zu Stunde. Das Ergebnis war das in Tab. VI auf S. 73 angegebene.

Diese Versuche (es wurden im ganzen acht Versuche an vier Personen gemacht) zeigen übereinstimmend, dass durch Pilokarpineinwirkung eine ganz deutliche Harnsäurevermehrung hervorgebracht wird, welche gleich in der ersten Stunde anhebt und etwa 3 Stunden andauert; nach zweifacher Pilokarpininjektion erscheinen dementsprechend auch zwei Maxima der Harnsäurevermehrung. Diese ist jedoch nicht so gross wie nach der Fleischeinnahme; die stündliche Harnsäuremenge steigt unter Pilokarpineinwirkung etwa von $25 \mathrm{mg}$ auf $40 \mathrm{mg}$, also um etwa $60 \%$, wogegen nach Fleischeinnahme dieselbe von $25 \mathrm{mg}$ auf $80 \mathrm{mg}$, also um mehr als $200 \%$ vermehrt wird. Doch ist die Harnsäurevermehrung durch Pilokarpin nicht unbedeutend, ihr zeitlicher Verlauf entspricht auch der angeregten Drüsentätigkeit wie nach der Fleischeinnahme;'in beiden Fällen beginnt die Harnsäurevermehrung sofort und schwindet in einigen Stunden. 
Der physiologische Protoplasmastoffwechsel und die Purinbildung.

Tabelle VI.

Einfluss des Pilokarpins auf die Harnsäureausscheidung.

\begin{tabular}{|c|c|c|c|c|c|c|}
\hline Anmerkung & $\begin{array}{l}\text { Zahl } \\
\text { des } \\
\text { Ver- } \\
\text { suches }\end{array}$ & $\begin{array}{l}\text { Stunden- } \\
\text { phase }\end{array}$ & $\begin{array}{l}\text { Harn- } \\
\text { menge } \\
\text { ccm }\end{array}$ & $\begin{array}{l}\text { Gesamt- } \\
\text { stickstoff } \\
\mathrm{g}\end{array}$ & $\begin{array}{c}\text { Harn- } \\
\text { säure } \\
\mathrm{g}\end{array}$ & $\begin{array}{c}\text { Anteil des } \\
\text { Harnsäure-N } \\
\text { am Gesamt-N } \\
\%\end{array}$ \\
\hline $\begin{array}{l}\text { Versuchspers. J, } \\
\text { 26. Febr. } 1887\end{array}$ & $\begin{array}{l}1 \\
2 \\
3 \\
4 \\
5 \\
6\end{array}$ & $\begin{array}{l}7-8 \mathrm{~h} \text { morg. } \\
8-9 \mathrm{~h} \\
9-10 \mathrm{~h} \\
10-11 \mathrm{~h} \\
11-12 \mathrm{~h} \\
12-1 \mathrm{~h}\end{array}$ & $\begin{array}{l}27 \\
37 \\
44 \\
47 \\
37 \\
40\end{array}$ & $\begin{array}{l}0,329 \\
0,468 \\
0,482 \\
0,496 \\
0,426 \\
0,459\end{array}$ & $\begin{array}{l}0,0208 \\
0,0270 \\
0,0313 \\
0,0317 \\
0,0277 \\
0,0275\end{array}$ & $\begin{array}{l}2,10 \\
1,92 \\
2,16 \\
2,12 \\
2,16 \\
1,99\end{array}$ \\
\hline $\begin{array}{c}\text { 1h } 30^{\prime} 0,012 \mathrm{~g} \\
\text { Pil, mur. }\end{array}$ & 7 & $1-2 \mathrm{l}$ & 36 & 0,278 & 0,0240 & 2,92 \\
\hline & $\begin{array}{r}8 \\
9 \\
10 \\
11\end{array}$ & $\begin{array}{l}2-3 h \\
3-4 h \\
4-5 h \\
5-6 h\end{array}$ & $\begin{array}{l}52 \\
39 \\
37 \\
30\end{array}$ & $\begin{array}{l}0,457 \\
0,429 \\
0,465 \\
0,398\end{array}$ & $\begin{array}{l}\mathbf{0 , 0 3 5 3} \\
\mathbf{0 , 0 3 9 3} \\
\mathbf{0 , 0 3 1 9} \\
0,0198\end{array}$ & $\begin{array}{l}2,58 \\
3,05 \\
2,29 \\
1,69\end{array}$ \\
\hline $\begin{array}{l}\text { Dieselbe Ver- } \\
\text { súchsperson, } \\
\text { 5. März } 1887\end{array}$ & $\begin{array}{l}1 \\
2 \\
3\end{array}$ & $\begin{array}{l}7-8 \mathrm{~h} \\
8-9 \mathrm{~h} \\
9-10 \mathrm{~h}\end{array}$ & $\begin{array}{l}62 \\
54 \\
55\end{array}$ & $\begin{array}{l}0,601 \\
0,554 \\
0,530\end{array}$ & $\begin{array}{l}0,0277 \\
0,0297 \\
0,0347\end{array}$ & $\begin{array}{l}1,58 \\
1,78 \\
2,18\end{array}$ \\
\hline $\begin{array}{c}10^{\text {h }} 45^{\prime} 0,01 \mathrm{~g} \\
\text { Pil. mur. }\end{array}$ & 4 & $10-11 h$ & 49 & 0,454 & 0,0280 & 2,05 \\
\hline $\begin{array}{c}11^{\mathrm{h}} 30^{\prime} 0,01 \mathrm{~g} \\
\text { Pil. mur. }\end{array}$ & 5 & $11-12 h_{1}$ & 60 & 0,409 & 0,0248 & 2,01 \\
\hline & $\begin{array}{r}6 \\
7 \\
8 \\
9 \\
10 \\
11\end{array}$ & $\begin{array}{r}12-1 \mathrm{~h} \\
1-2 \mathrm{~h} \\
2-3 \mathrm{~h} \\
3-4 \mathrm{~h} \\
4-5 \mathrm{~h} \\
5-6 \mathrm{~h}\end{array}$ & $\begin{array}{l}57 \\
54 \\
33 \\
45 \\
37 \\
41\end{array}$ & $\begin{array}{c}0,445 \\
0,462 \\
0,424 \\
0,557 \\
\overline{4448}\end{array}$ & $\begin{array}{l}\mathbf{0 , 0 3 8 3} \\
\mathbf{0 , 0 4 7 9} \\
\mathbf{0 , 0 3 9 8} \\
\mathbf{0 , 0 4 5 1} \\
0,0321 \\
0,0310\end{array}$ & $\begin{array}{l}2,84 \\
3,45 \\
3,12 \\
2,71 \\
\overline{2,30}\end{array}$ \\
\hline
\end{tabular}

Durch diese Versuche erschien mir als erwiesen, dass die Tätigkeit der Verdauungsdrüsen mit Harnsäurebildung verbunden ist. In Anbetracht der histologischen Tatsache, dass bei dieser Tätigkeit sehr eingreifende materielle Veränderungen im Protoplasma der Drüsenzellen vor sich gehen, durch welche die spezifischen Verdauungssekrete gebildet werden, kam ich zu der Überzeugung, dass eben dieser physiologische Stoffwechsel im Protoplasma eine Quelle der Harnsäure ist. Auf Grund dieser Erkenntnis gelangte ich zur allgemeinen Fasșung der Theorie, dass die Harnsäure ein Produkt des physiologischen Stoffwechsels im Protoplasma der Körperzellen ist, $z u$ welcher Verallgemeinerung die Tatsache der individuellen Konstanz der Harnsäure im nüchternen Zustande ein zureichender Grund ist. „So hätten wir in der Harnsäure ein Maass des physiologischen Stoffwechsels im Protoplasma." 
„Dieser Theorie gelang es nicht, sich allgemeine Anerkennung zu gewinnen", bemerkten Burian und Schur ${ }^{1}$ ). Denn die späteren Untersuchungen und Anschauungen in dieser Frage liessen sich von bloss chemischen Gesichtspunkten leiten, so dass der erkannte chemische Zusammenhang der Harnsäure mit dem Nuklein die eigentlich physiologische Seite der Frage zuerst mehr verdunkelte als beleuchtete.

Als dann die Unzulänglichkeit einer bloss chemischen Betrachtung physiologischer Fragen auch auf diesem Gebiete erkannt worden war und die physiologische Seite des Ursprungs der Harnpurine, dank den Arbeiten von Sivén und von Burian mit Schur, wieder in den Vordergrund trat, da wurde die Aufmerksamkeit auch auf meine Theorie gelenkt. Nun wurden aber gerade gegen den tatsächlichen Grund derselben, nämlich gegen den Zusammenhang der Harnsäurebildung mit der Tätigkeit der Verdauungsdrüsen, Einwände erhoben, so dass nur der ganz alligemeine Satz daraus übrig blieb, dass die Harnsäure ein Produkt der Körperzellen sei.

Diese Sachlage hatte mich bestimmt, den tatsächlichen Grund meiner Theorie hier von neuem darzulegen, und führt mich jetzt zu einer kritischen Untersuchung jener gegen denselben vorgebrachten Einwände, um die Berechtigung meiner Theorie darzutun.

III Kritische Untersuchung der Frage eines Zusammenhanges
der Harnsäurebildung mit der Tätigkeit der Verdanungsdrüsen.

"Die Theorie von Mares", berichtet Wiener ${ }^{2}$ ), "weist aber eine grosse Lücke auf, dadurch nämlich, dass sie den Einfluss des Nahrungsnukleins gar nicht berücksichtigt. Zu der Zeit, wo diese Theorie aufgestellt wurde, war diese Unterlassung entschuldbar, da bis dahin sämtliche Fütterungsversuche mit Nukleinen oder Pưrinkörpern ein negatives Frgebnis geliefert haben, bis auf einen Versuch Horbaczewski's...."

Aber auch dieser Versuch ist erst 4 Jahre nach Aufstellung meiner Theorie veröffentlicht worden; meine Schuld, Entdeckungen nicht berücksichtigt zu haben, die erst später gemacht worden sind, erklärt sich aus der allgemeinen menschlichen Schwäche in dieser Richtung. Diese allgemein menschliche Verschuldung war aber auch

1) Burian und Schur, Pflüger's Arch. f. d. ges. Physiol. Bd.80 S. 252.

2) H. Wiener, Ergebn. d. Physiol. Bd. 1 (1) S. 579. 1902. 
in diesem Falle verhängnisvoll. Die Beziehung der Nukleine und der Xanthinkörper zur Harnsäure ist; nach deren Entdeckung durch Ho rbaczewski, der Ausgangspunkt ziemlich aller Untersuchungen auf diesem Gebiete geworden, und man wurde geneigt, die Harnsäure überhauṕt von den Nahrungspurinen und Nukleinen herzuleiten und jede Beziehung derselben zum Nahrungs e i weiss abzulehnen. We intraud ${ }^{1}$ ) fand nach Einnahme von Kalbsthymus eine sehr grosse Vermehrung der ausgeschiedenen Harnsäure, mit parallel vermehrter Phosphorausscheidung, während der Gesamtstickstoff des Harns verhältnismässig wenig zugenommen hatte; wurde anstatt Thymus Muskelfleisch zur gewöhnlichen Kost zugeg eben, so viel, dass eine gleiche Vermehrung des Gesamtstickstoffes erfolgte, so hatte diese Zulage auf die Harnsäuremenge k e in en Einfluss.

Dieses Ergebnis schien darauf zu deuten, dass die Harnsäurebildung vom Nukleingehalte der Nahrung beeinflusst wird, wenig oder gar nicht aber von deren Hiweissgehalte. In der Tat haben gleich darauf $\mathrm{Hes}$ e und Schmol1 ${ }^{2}$ ) nach Zulage von purinfreiem Eiweiss (aus 24 Eiern) zur gewöhnlichen Fleischkost keine Vermehrung der Harnsäure und der Alloxurkörper im Harne gefunden und sahen in diesem Versuche den "strikten Beweis für das vollkommene Fehlen eines Zusammenhanges zwischen Eiweissnahrung und der Harnsäure und die Berechtigung, in den Nukleinen und den Alloxurkörpern der Nahrung eine direkte Quelle der Harnsăure im Organismus zu sehen". Ebenso behauptete J. S. Jerome ${ }^{3}$ ) die grosse Abhängigkéit der ausgeschiedenen Harnsäuremenge von den Nukleinen und Alloxurkörpern der Nahrung, wenn auch andere - Finflüsse nicht auszuschliessen seien; die fortwährende Harnsäureausscheidung, wenn auch in geringerem Maasse, während längeren Hungerns sei dem Katabolismus der nukleinhaltigen Zellen zuzusehreiben.

Gègen die Herkunft der Harnsäure aus den Nahrungs-Nukleinen haben zunächst $\mathrm{Hopkins}$ und $\mathrm{H}_{0} \mathrm{pe}^{4}$ ) folgenden Finwand erhoben. Sich auf das Ergebnis meiner. Untersuchungen berufend, dass näm-

1) Weintraud, Arch. f. Physiol. 1895 S. 382 .

2) Hess und Schmoll, Arch. f. exper. Pathol. u. Pharmakol. Bd. 37 S. 243.1896.

3) J. S. Jerome, The Journ. of Physiol. vol. 22 p. 146. 1897; vol. 25 p. 98.1899.

4) Hopkins and Hope, The Journ. of Physiol. vol. 28 p. 271. 1898. 
lich nach Fleischeinnahme die Vermehrung der Harnsäure früher eintritt und vorübergeht als die Vermehrung des Gesamtstickstoffes, fanden sie es merkwürdig, dass ein Produkt eines durch den Magensaft am schwersten verdaulichen Proteids der Nahrung am raschesten zur Ausscheidung gelangen sollte. Deswegen wiederholten Hopkins und Hope meine diesbezüglichen Versuche und fanden dieselben vollständig bestätigt: Die Harnsäurevermehrung nach Einnahme nukleinhaltiger Nahrung (Fleisch, Thymus) tritt viel früher ein als die Vermehrung des Harnstoffes. Daraus schlossen sie, dass diese Harnsäure nicht von dem Nahrungsnuklein herstammen könne, sondern von anderen Nahrungsstoffen. Denn Einnahme von reinem Nuklein bewirke keine ganz konstante und keine so sichere und grosse Harnsäurevermehrung wie die Einnahme von Fleisch, welches verhältnismässig arm an Nuklein ist. Ein aus durch künstlichen Magensaft verdauter Thymus bereitetes Extrakt, das wohl frei von Nuklein ist, bewirkt nach der Einnahme eine rasche und abundante Harnsäurevermehrung. Die Harnsäurevermehrung nach Fleisch- oder Thymuseinnabme wollten Hopkins und Hope auch nicht mit dem Eiweiss dieser Nahrungsmittel in Beziehung bringen, weil sie nach Eiweissund Milcheinnahme eine viel geringere Harnsäurevermehrung beobachteten als nach Fleischgenuss. Den harnsäurevermehrenden Einfluss von Fleisch und Thymus brachten sie also nicht mit deren Nuklein, sondern mit anderen durch Magenverdauung in Lösung gelangenden Stoffen in Zusammenhang. Jerom $\mathrm{e}^{1}$ ) wies dabei auf die Xanthinkörper oder Alloxurbasen des Fleisches und der Thymus als die eigentlichen Muttersubstanzen der Harnsäure hin.

Tatsächlich fand dann Si vén ${ }^{2}$ ), dass gerade die Extraktivstoffe des Fleisches, welche auch im Bouillon enthalten sind, die Harnsäurevermehrung bewirken, während das Eiweiss selbst durchaus keinen Einfluss auf die Harnsäureausscheidung hat; diese hält sich vielmehr für jeden Tag hartnäckig um denselben Wert, unabhängig davon, ob die Kost viel oder wenig Eiweiss enthält. Ein Teil der ausgeschiedenen Harnsäure stammt, nach Sivén, direkt von den eingenommenen Purinbasen, indem dieselben direkt zur Harnsäure oxydiert werden; der andere Teil stammt aus dem Körper selbst, aus den eigenen Zellen, und dieser Teil ist von der Nahrung (in-

1) Jerome, The Journ. of Physiol, vol. 25 p. 98. 1899.

2) V. O. Sivén, Skand. Arch. f. Physiol. Bd. 11 S. 139.1901. 
sofern dieselbe purinfrei ist) vollständig unabhängig. Si vé $\mathbf{n}^{1}$ ) reproduzierte einen meiner Versuche über die Harnsäurevermehrung nach Fleischeinnahme, auf Grund deren zeitlichen Verlaufes ich die Harnsäurebildung mit der durch die Nahrung angeregten Tätigkeit der Verdauungsdrüsen in Zusammenhang gebracht habe. Dabei erledigte er aber diese Frage folgendermaassen: „Da man jedoch nunmehr weiss, dass die Extraktivstoffe des Fleisches allein die Harnsäureausscheidung bedeutend und ziemlich rasch vermehren, so ist wohl die Erklärung richtiger, dass es gerade diese Stoffe im Fleische sind, welche die rasche Vermehrung verursachen, indem sie direkt zu Harnsäure oxydiert werden, ohne organisiert zu werden."

Damit erschien meine Theorie als abgeschafft, ja gerade ihr Gegenteil wurde als Tatsache hingenommen. Da z. B. S i én während der Zeit der lebhaftesten Verdauungsarbeit die Harnsäureausscheidung teils grösser, teils geringer fand als während der Verdauungsrube, sah es ihm so aus, als ob die Digestionsarbeit keinen Einfluss auf die Harnsäurebildung ausübte. Nun fragte sich aher Sivên selbst, durch welchen vitalen Prozess die Harnsäuremenge gebildet wird, welche aus dem Organismus selbst herstammt. . Nach den Untersuchungen Kossel's", antwortete er, „wäre zunächst daran zu denken, dass die Harnsäure aus dem Nukleinstoffe der Zellkerne erzengt wurde, wodurch die Harnsäure (wie überhaupt die Purinbasen) als ein Stoffwechselprodukt innerhalb des Zellenkernes selbst anzusehen wäre. Ob der Zellkern der einzige Herd der Harnsäurebildung ist, und ob dieser Prozess in alle Zellen des Organismus zu verlegen ist oder nur in einen Teil derselben, das sind Fragen, die noch immer ihrer Lösung harren". Sivén hatte nämlich in erster Linie gegen die Leukocytosetheorie Stellung genommen und es höchst unwahrscheinlich gefunden, dass die Harnsäure unter normalen Verhältnissen aus den Nukleinstoffen der Leukocyten gebildet wird, ohne dies jedoch ganz zu verneinen. Da ihm nun auch die Drüsenarbeit die Harnsäurebildung nicht zu verstärken schien, so blieben ihm schliesslich die Muskelzellen als innere Quelle der Harnsäure übrig, wofür ihm der Unterschied in der Harnsäureproduktion während des Tages und der Nacht, entsprechend dem Unterschiede in der Muskeltätigkeit, zu sprechen schien; später aber

1) V. O. Sivén, Skand. Arch. f. Physiol. Bd. 11 S. 146. 1901, 
schloss er $^{1}$ ), gegenüber Burian ${ }^{2}$ ), äuch diese Quelle aus und bezeichnete die Nierentätigkeit als innere Quelle der Harnsäure. So ist denn zuletzt Sivén selbst auf die Drüseṇtätigkeit als Quelle der Harnsäure zurückgekommen. Der tatsächliche Grund, aus welchem Sivén den Zusammenhang der Harnsäurebildung mit der Tätigkeit der Nieren anerkannte, den Zusammenhang mit der Tätigkeit der Verdauungsdrüsen aber ablehnte, wird später, in Verbindung mit anderen derselben Art, von mir untersucht werden.

Burian und Schur ${ }^{3}$ ) haben gleichzeitig mit Sivén die e ndogene Harnsäurequelle von der ex ogenen zu unterscheiden und zu bestimmen' gesucht. Auch sie betrachteten die Nahrungspurine als direkte Muttersubstanzen der Harnpurine und hielten daran fest, dass purinfreie Nahrung im menschlichen Stoffwechsel weder Harnsäure noch Xanthinbasen liefert, so dass die Kost des Menschen ausser den Purinen keine Stoffe" enthält, welche die Harnpuriubildung beeinflussen und ihre Ausscheidung alterieren. Dafur beriefen sie sich besonders auf die Versuche von Hess und Schmoll Von diesem Standpunkt. aus versuchten Burian und Schur den endogenen Harnsäurewert zu bestimmen, als welchen sie aber nicht deren Hungerwert gelten lassen wollten, wie ich denselben im nüchternen Zustande bestimmt habe. Sie fanden es nicht richtig, den "Hungerwert" der Harnsäureausscheidung eines Individuums mit dem endogenen Anteile seiner Harnsăureausfuhr bei zureichender Ernährung zu identifizieren, weil im Hunger der ganze Ablauf des Stoffwechsels zweifellos sehr stark alteriert wird, da hier abnorme Alloxurkörperquellen in Tätigkeit treten, indem eine Einschmelzung: xanthinbasenhaltigen Materials (Muskel usw.) Platz greift, so dass der Harnsäurehungerwert dann grösser wäre als der endogene Harnsäureanteil desselben Individuums unter den gewöhnlichen Ernäbrungsbedingungen; andererseits könnte aber im Hungerzustande die endogene Harnsäurebildung auch eingeschränkt sein. Diese letztere Möglichkeit bezeichnen B urian und S ch ur als den Tatsachen wirklich entsprechend, da Ranke und K. B. Hofmann die Harnsäureausfuhr nach Genuss von vollständig stickstofffreier Kost grösser gefunden haben als im Hunger.

1) V. O. Sivén, Skand. Arch. f. Physiol. Bd. 18 S. 193. 1906.

2) R. Burian, Zeitschr. f. physiol. Chemie Bd. 43 S. 532.19041905.

3) R. Burian und H. Schur, Pflüger's Arch. f. d. ges. Physiol. Bd. 80 S. 241. 1900 . 
Dessenungeachtet unternahmen es Burian und Schur, den endogenen Harnsäurewert bei zureichender Ernährung mit purinfreier Kost zu bestimmen, unter der festen Voraussetzung, dass eine solche Kost keinen Einfluss auf die endogene Harnsäurebildung ausübe, da die endogenen Harnpurine aus Prozessen stammen, welche relativ unabhängig sind von dem Ausmaasse und der Zusammensetzung der Nahrung. Die Bestimmung geschah in der Weise, dass die flejschreiche gewöhnliche Diät, mit der sich die Versuchspersonen im Stickstoffgleichgewicht befanden, durch purinfreie Diät ersetzt wurde, wobei die Harnsäuremenge auf den rein endogen Wert herabsank, welcher sich für ein und dasselbe Individunm als eine ph y si 0 logische Konstante zeigte.

Burian und Schur suchten wohl anch die Voraussetzung dieser Bestimmung zu befestigen, dass nämlich purinfreie Nahrung keinen Einfluss auf die endogene Harnsäurebildung ausübe. So fänden sie die endogene Harnpurinmenge absol ut unabhängig von der (purinfreien) Nahrung, da dieselbe konstant bleibt selbst bei grossen Schwankungen der Kost; dann erklärten sie aber dieselbe für recht unabhängig, für innerhalbweiter Grenzen unabhängig, um zuletzt, in der Diskussion mit Loewi, eine gewisse Abhängigkeit der endogenen Harnsäurebildung von der Nahrungsaufnahme doch zuzugeben. "Gibt man eine sehr abundante, den Darm mächtig beanspruchende Nahrung, so dass (durch die Verdauungsarbeit) der "Kalorienumsatz" wesentlich erhöht ist, so stejğt die endogene Harnpurinmenge an; ist die gereichte Kost hinwiederum unzureichend, so dass der Organismus allmählich den Gesamtumsatz einschränkt, so nimmt die endogene Harnpurinmenge $a b$, um endlich im vollen Hunger beträchtlich geringer zu sein als bei zureichender Ernähruag mit nahrungspurinfreier Kost " ${ }^{1}$ ). Damit ist zugestanden, dass purinfreie Nahrung die endogene Harnsäurebildung anregen kann; und zwar durch Inanspruchnahme der Verdauungsarbeit.

Die Verknüpfung der endogenen Harnsäurebildung mit dem „Kalorienumsatze“, ebenso wie die Erwähnung der Einschmelzung der Organe im Hungerzustande wurde von Burian und Schur wohl nur als eine Bemerkung ohne tatsächliche. Bedeutung gedacht. Diese Bemerkung wurde aber in einem solchen Sinne hingenommen, wodurch ein Missverständnis entstanden ist, welches $B$ u ri a n und

1) Burian und Schur, Pflüger's Arch. Bd. 80 S. 342. 
Schur eigentlich selbst berichtigen sollten. Man wurde nämlich ganz misstrauisch gegen Versuche, welche im „Hungerzustand" bei "ungenügender Kalorienzufuhr" gemacht worden sind. Sehr genau nabmen die Sache z. B. Kaufmann und Mohr ${ }^{1}$ ), als sie „berechtigte Bedenken" gegen Versuche aussprachen, die vom Hunger bzw. ungenügender Kalorienzufuhr ausgingen, welches Verfahren unzulässig sei, „wie wir jetzt wissen“. Der "Hungerzustand“ ist aber der sicherste Ausgangspunkt bei Stoffwechsel- und Ernährungsversuchen, weil hier der störende Einfluss der Nahrungsaufnahme eliminiert ist und der Stoffwechsel sehr gleichmässig verläuft. Wohl darf dieser Zustand nicht ein so hochgradig fortgeschrittener sein, wo es schon zum Einschmelzen der Organe und Einschränken des "Kalorienumsatzes" kommt, sondern ein solcher, in welchem der Organismus von seinen eigenen reichlichen Nahrungsreserven lebt, wie er es auch bei regelmässiger Nahrungsaufnahme tut, welche zunächst zur Ersetzung der eigenen verbrauchten Reserven dient. Vom "Kalorienumsatz" ohne ein Kalorimeter zu reden ist sehr bedenklich. Wenn sich $\mathrm{B}$ urian und $\mathrm{S}$ ch ur wegen des erhöhten Kalorienumsatzes bei der Verdauungsarbeit auf $R \mathfrak{a b n e r}$ berufen haben, so übersahen sie die Versuche desselben Autors über Isodynamie der Nahrungsstoffe, wo es heisst: ${ }^{2}$. Es muss hier auch die täglich produzierte Wärmemenge die gleiche geblieben sein, ob Eiweiss gefüttert wurde, oder ob sich der Körper im Hungerzustand befand" ${ }^{2}$ ). Doch dieses sind ganz andere, einer kritischen Erörterung bedürftige Fragen.

$\mathrm{Ka} \mathrm{ufmann}$ und $\mathrm{Mohr}^{3}$ ) beschäftigten sich unter v. Noorden's Leitung ebenfalls mit der Frage, ob der nach nukleinfreier Kost zu erzielende endogene Alloxurwert ein konstanter Wert sei, oder aber, ob er sich durch Änderung der Kostordnung beeinflussen lässt. Dazu haben sie die Versuchsperson auf eine konstante nukleinfreie Kost gesetzt und untersuchten, „ob Zulage einer grossen Kalorienmenge den endogenen Wert beeinflusst". So prüten sie $\mathrm{Zulagen}$ von Fett und Kohlenhydraten und prüften auch die Versuche von Hess und Schmoll nach, d. i. Zulagen von grossen Mengen nukleinfreien Eiweisses, „zumal jene Autoren mit der ungenauen Methode von Krüger-Wulff gearbeitet haben“. Ihr Ergebnis aber war

1) Kau fmann und Mohr, Deutsches Arch. f. klin. Medizin Bd. 74 S. 141. 1902.

2) M. Rubner, Zeitschr. f. Biol. 1883 S. 348.

3) Kaufmann und Mohr, Deutsches Arch. f. klin. Med. Bd. 74 S. 141. 1902. 
ein gleiches: "Nukleinfreie Eiweisszulagen beeinflussen die Alloxurund Harnsäureausscheidung jedenfalls nicht wesentlich. Eine ganz geringfügige Erhöhung kann stattfinden, ist jedoch so gering, dlass sie sich ungezwungen aus der Mehrbelastung des Darms (nach Burian und Schur) erklären lässt. Wir können also die Befunde von Hess und Schmoll mit einwandfreier Methode bestätigen." Kaufmann und Mohr legen auf die Genauigkeit der Methoden grosses Gewicht; wir werden sehen, inwiefern chemisch genaue Methoden den Erfolg physiologisch ungenauer Versuche verbürgen.

Der Satz, dass purinfreie Nahrung, besonders Eiweiss, keinen Einfluss auf die Harnsäureausscheidung ausübt, wurde so allmählich als sichergestellt angenommen. Pfeil ${ }^{1}$ ) drückte dies mit diesen Worten aus: "Der Fortschritt der Erkenntnis besteht darin, dass der Eiweissgehalt der Nahrung für die Grösse der Harnsäureausscheidung definitiv als einflusslos festgelegt ist." Diesen Fortschritt förderte Pfeil durch weitere Versuche, in welchen bei fleischfreier, dreimal täglich eingenommener Nahrung die in dreistündigen Perioden bestimmte Harnsäuremenge keinen Einfluss der Nahrung zeigte, so dass sich der Mensch auf ein bestimmtes Quantum der Harnsäuremenge stellte, welches auch durch stickstofffreie Kost nicht herabgedrückt wurde, während Einnahme von Fleisch einen sofortigen Anstieg desselben bewirkte.

Das Ergebnis aller dieser Untersuchungen wird nun allgemein so aufgefasst, dass purinfreie Nahrung überhaupt keinen Einfluss auf die endogene Harnsäurebildung ausübt; und dadurch ist die von mir vertretene Theorie, dass die durch die Nahrung angeregte Tätigkeit der Verdauungsdrüsen eine Quelle der endogenen Harnsäure bildet, als widerlegt ausser Betracht gekommen. Deswegen finde ich mich veranlasst, jene Untersuchungen sowie die aus ihnen gezogenen Schlüsse einer kritischen Betrachtung zu unterziehen.

Wir haben es hier mit zwei ganz verschiedenen Fragen zu tun, die klar auseinanderzuhalten sind. Die erste, uns eigentlich interessierende Frage ist: ob die eingenommene Nahrung, besonders das Eiweiss, eine Vermehrung der ausgeschiedenen Harnsäure bewirke, und zwar als ein die Tätigkeit der Verdauungsdrüsen anregender Reiz. Die zweite Frage aber ist eine ganz andere: ob ein Verhältnis bestehe zwischen der Menge des Nahrungseiweisses und der Menge der ausgeschiedenen Harnsäure.

1) P. Pfeil, Zeitschr. f. physiol. Chemie Bd. 40 S. 1. 1903 
Alle angeführten Untersuchungen sind so angestellt, dass sie eine Antwort nur auf die zweite, nicht aber auf die erste Frage enthalten können. Es wurde nämlich, bei gleichmässiger Ernährung, entweder die Menge des purinfreien Nahrungseiweisses variiert (Sivén, Burian und Schur), oder es wurden zur gewöbnlichen Kost grosse Zulagen von purinfreiem Eiweiss oder von anderen Nahrungsstoffen gemacht (Hess und Schmoll, Kaufmann und $\mathrm{Mohr}$ ). Das Ergebnis war übereinstimmend, dass die ausgeschiedene Harnsäuremenge in keinem Verhältnis zu der eingenommenen Eiweissmenge steht, da sie bei grossen Variationen dieser konstant bleibt. Wenn also z. B. Pfeil sagt, es sei als definitiv festgelegt zu betrachten, dass der Eiweiss gehalt der Nahrung keinen Einfluss auf die ausgeschiedene Harnsäuremenge hat, so hat er ganz recht. Aber dieser Satz ist gar nicht neu, er ist aus meiner Tabelle I ganz klar zu ersehen: Die Harnsäuremenge ist individuell konstant, bei sehr grossen Variationen des ausgeschiedenen Gesamtstickstoffes, welche durch Variationen des Eiweissgehaltes der 12 Stunden vor Beginn jedes Versuches eingenommenen Nahrung bewirkt wurden. Jene Untersuchungen haben also den Wert überzähliger Beweise einer Tatsache, die viel früher schon den Ausgangspunkt weiterer Nachforschungen gebildet hat. Und diese Nachforschungen betrafen zunächst unsere erste Frage.

Nun wird aber das Ergebnis jener Untersuchungen von ihren Autoren auch auf diese unsere erste Frage ausgedehnt. So schliessen Hess und Schmoll: Diese Versuche bilden einen strikten Beweis für das vollkommene F'ehlen eines Zusammenhanges zwischer Eiweissnahrung und Harnsäure. Si vén folgert aus seinen Versuchen richtig, dass die Eiweiss menge der Nahrung auf die Harnsäure nicht einwirkt, erweitert aber zuletzt diese Folgerung so, dass die Kost, wenn sie nicht Purinstoffe enthält, durchaus keinen Einfluss auf die Harnsäureausscheidung ausübt, und schliesst demnach die Tätigkeit der Verdauungsdrüsen als innere Quelle der Harnsäure gauz aus. Burian und Schur finden ebenfalls die Harnsäuremenge unabhängig von der eingenommenen purinfreien Fiweissmenge, schliessen aber doch, dass die Harnsäuremenge von der Nahrung (überhaupt) absolut unabhängig ist oder wenigstens recht unabhängig, in weiten Grenzen unabhängig, insofern die Nahrung nicht viel Verdauungsarbeit in Anspruch nimmt. Thnen folgen Kaufmann und Mohr. 
Die Versuchsanordnung dieser Untersuchungen aber ist eine solche, dass dieselben auf unsere erste Frage gar keine Antwort geben können; denn sie besteht nur in Variationen der M enge vornehmlich des Eiweisses in der regelmässig eingenommenen an sich schon eiweisshaltigen Nahrung. Fragt es sich also, ob die durch die eingenommenen Nahrungsstoffe als Reize angeregte Tätigkeit der Verdauungsdrüsen mit vermehrter Harnsäureausscheidung verbunden ist oder nicht, so ist darauf aus solchen Versuchen keine Autwort zu bekommen. Denn erstens, das quantitative Verhältnis zwischen einem Reize und der durch denselben angeregten physiologischen Reaktion ist kein festes und ist durch ein Minimum und ein Maximum begrenzt; durch supramaximale Reize wird keine grössere Reaktion mehr hervorgerufen. Gerade bei der Anregung der Tätigkeit des Verdauungsorgans durch die Nabrungsstoffe kommt es vielmehr auf deren Qualität als deren Quantität an. Übermässig grosse Nahrungsmengen brauchen keine erhöhte Verdauungsarbeit anzuregen, sondern können einfach im Darmkanal liegen bleiben. Und weiter, die angeregte Tätigkeit besteht hier zunächst in der Bildung der Verdauungsfermente; diese aber, einmal gebildet, vermögen sehr grosse Mengen von Nahrungsstoffen zu verarbeiten, denn die Mengenverhältnisse zwischen den Fermenten und ihren Substraten sind sehr weite. Wenn also nach Vermehrung der Eiweissmenge in der gewöhnlichen eiweisshaltigen Kost keine Vermehrung der ausgeschiedenen Harnsäure eintritt, so kann daraus nicht geschlossen werden, dass die Harnsäurebildung mit der Tätigkeit der Verdauungsdrüsen nicht zusammenhängt, weil vermehrte Eiweissmenge in der Nahrung keine vermehrte Drüsentätigkeit hervorzurufen braucht. Wäre die Menge der ausgeschiedenen Harnsäure von der Menge des eingenommenen Eiweisses abhängig, so wäre es bei der alten Ansicht geblieben, dass die Harnsäure eine Vorstufe des Harnstoffes ist. Ich habe vor 23 Jahren festgestellt, dass die Harnsäuremenge von der. Menge des Nahrungseiweisses ganz unabhängig ist, und das gerade bestimmte mich, nach ihrem Ursprung im "Protoplasma" der Zellen, zunächst der Drüsenzellen, zu suchen. Burian und $\mathrm{S}$ chur ${ }^{1}$ ) haben aus meiner damaligen Abhandlung das Wort "Organeeiweiss" aufgegriffen, und $\mathrm{W}$ iene ${ }^{2}$ ) fasste dies so auf, dass ich „gewisser-

1) Burian und Schur, Pflüger's Arch. f. d. ges. Physiol. Bd. 80 S. 252.1900.

2) H. Wiener, Ergebnisse der Physiologie Bd. 1 (1) S. 582.1902. 
maassen " die alte Anschauung von der Herkunft der Harnsäure a u s dem Eiweisse wieder aufnahm. Das wäre aber ein grosses Missverständnis, verschuldet vielleicht durch den damals von mir gebrauchten Ausdruck "albumine organisée", welchen ich im Sinne von "Protoplasma" angewendet habe, das wohl mit. „Eiweiss" im chemischen Sinne gar nicht $z u$ verwechseln ist.

Zweitens, ein Zusammenhang zwischen Harnsäurebildung unil der durch die Nahrung angeregten Tätigkeit der Verdauungsdrüsen kann n i c bt g'efunden werden, wenn diese Tätigkeit durch regelmässige, dreimal täglich erfolgende Nahrungsaufnahme gleichsain in Permaneuz gehalten wird, in der Meinung, dass dieselbe durch grosse Z $11-$ lagen von bestimmten Nahrungsstoffen zu dieser gewöhnlicben host zur grösseren Intensität gebracht werden kann, usid wenn die Har'ısäureausscheidung dabei in 24 stündigen Perioden bestimmt wird, wo die voraussichtlichen stündlichen Variationen derselben vollständig verschwinden müssen. Wird auf Grund soleher Untersuchuagen jeder Zusammenhang zwischen der Drüsentätigkeit und der Harnsäurebildung bestritten, so ist es ein Fehlschluss; denn in solchen Versuchen kann sich dieser Zusammenhang überhaupt nicht zeige n. Auch hier schloss nur Pfeil ${ }^{1}$ ) ganz richtig: „Der Einfluss der Nahrung hat keinen Erfolg auf die Harnsäuremenge"; das ist wohl so zu verstehen, dass dieser Erfolg hier nicht bemerkt werden kann.

Die Versuchsanordnung zur Löung der Frage, ob die durch Nahrung als Reiz angeregte Verdauungsdrüsentätigkeit mit Harnsäurebildung verbunden ist, ergibt sich von selbst aus allgemeinen physiologischen Prinzipien. Die Harnsäureausscheidung ist währeud der Ruhe und während der Tätigkeit der Verdauungsdrüsen in ganz kurzen, womöglich stündlichen Perioden zu bestimmen; der diese Tätigkeit anregende Reiz muss isoliert in einem bestimmten Zeitmoment die ruhenden Drüsen treffen. Eine solche Versuchsanordnung benutzte ich auch bei meinen Untersuchungen; ich verfolgte die Harnsäureausscheidung im nüchternen Zustande von Stunde zu Stunde und liess den die Drüsentätigkeit auregenden Reiz (Fleisch, Pilokarpin) zu einer bestimmten Stunde einwirken. Buria $\mathbf{n}^{2}$ ), der diese Methode des "Hungerzustandes" früher mitbemängelt hatte, fand

1) P. Pfeil, Zeitschr. f. physiol. Chemie Bd. 40 S. 10. 1903.

2) R. Burian, Zeitschr. f. physiol. Chemie Bd. 43 S 534. 1904-1905. 
dieselbe zuletzt doch vorteilbaft zur Untersuchung des Finflusses der Muskelarbeit auf die Harnpurinausscheidung.

Auf diese Weise fand ich, dass die Fleischeinnahme eine bedeutende, rasch ansteigende und bald vorübergehende Vermehrung der Harnsäureausscheidung bewirkt, während die danach folgende Vormehrung des gesamten Harnstickstoffes später erscheint und viel läıger andauert. Entsprechend diesem von Stunde zu Stunde verfoloten zeitlichen Verlaufe dieser Ausscheidungen brachte ich die Harnsäurevermehrung mit der angeregten Tätigkeit der Verdauungsdrüsen, die Vermehrung des Gesamtstickstoffes mit der Verdauung und Absorption des eingenommenen Eiweisses in Zusammenhang. Nun stehe ich vor dem Einwande, dass diese Harnsäurevermehrung nicht mit der Drüsentätigkeit, sondern mit den eingenommenen Fleischpurinen im Zusammenhange ist, welche als direkte Muttersuhstanzen der Harnsäure zu betrachten sind (Sivén, Burian und Schur).

Zur Behebung dieses Einwandes ist vor allem ein tatsächlicher Grund nötig. Es ist mit derselben Methode des nüchternen Zustandes zu untersuchen, ob auch purinfreies Eiweiss und purinfreie Nahrung überhaupt eine zeitlich ebenso verlaufende Harnsäurevermehrung bewirkt, in dem Maasse, als dadurch die Tätigkeit der Verdauungsdrüsen angeregt wird. Die Ergebnisse dieser neuen Untersuchungen werden im folgenden Artikel mitgeteilt werden.

Hier seien vorerst andere Untersuchungen dieser Art betrachtet. Hopkins und Hope ${ }^{1}$ ) haben, wie erwähnt, meine Untersuchungen über die Harnsäurevermehrung nach Fleischeinnahme mit der gleichen Methode wiederholt und bestätigt. Aber sie machten nebenbei auch zwei Versuche mit purinfreiem Eiweiss. Nach Einnahme einer grösseren Menge von Eiereiweiss und Milch, zu einer bestimmten Stunde des nüchternen Zustandes, erfolgte gleich in der ersten und zweiten Stunde eine Harnsäurevermehrung von $22 \mathrm{mg}$ auf 32 und $38 \mathrm{mg}$, welche Vermehrung dann ziemlich rasch auf den Wert des nüchternen Zustandes wieder zurückging. Aber Hopkins und Hope schien diese Vermehrung, im Vergleiche mit derjenigen nach Fleischoder Thymuseinnahme, sehr klein und unbedeutend, so dass sie auf diesen Befund gleichsam verzichteten. Doch in unserer Frage hat derselbe wohl eine Bedeutung. Burian ${ }^{2}$ ) hat, mit der gleichen

1) Hopkins and Hope, The Journ. of Physiol. vol. 23 p. 289.1898.

2) R. Burian; Zeitschr. f. physiol. Chemie Bd. 43 S. 534. 1904-1905. 
Methode des nüchternen Zustandes, nach einstündiger Muskelarbeit eine Vermehrung der Harnsäure von $11,4 \mathrm{mg}$ auf $18,2 \mathrm{mg}$ (um $60 \%$, in einem anderen Versuche von $7,3 \mathrm{mg}$ auf $22,2 \mathrm{mg}(200 \%)$ per Stunde gefunden, was ihm genügte, um die Harnsäurebildung mit der Muskeltätigkeit in Zusammenhang zu bringen (ogleich die stündlichen Werte der Harnsäure im nüchternen Zustande in seinen Versuchen ziemlich niedrig erscheinen). Ebenso könnte auch der Befund von Hopkins und Hope genügen, um die Harnsäurebildung mit der Tätigkeit der Verdauungsdrüsen zu verknüpfen.

Es fragt sich nun, warum die Einnahme von Fleisch eine viel grössere Harnsäurevermehrung bewirkt als die Einnahme von purinfreiem Eiweiss? Diese Frage wird vielleicht denjenigen nüssig erscheinen, welche es als festgestellte Tatsache hinnehmen, dass ja die Fleisch purine direkte Muttersubstanzen dieser Harnsäure und ihre exogene Quelle sind. Ich möchte aber hier gegen die Vertreter dieser Ansicht einen Gegeneinwand erheben und auf sie die Last eines Beweises durch tatsächliche Gründe überwälzen.

Aus den Untersuchungen Pawlow's ${ }^{1}$ ) und seiner Schule geht bervor, dass verschiedene Nahrungsmittel und Nahrungsstoffe die Arbeit der Verdauungsdrüsen in sehr ungleichem Grade anregen, und zwar je nach dem, wie es zu ihrer Verdauung nötig ist. So wird die Sekretion des Magensaftes durch einfache Proteinstoffe, wie z. B. koaguliertes Eiereiweiss, Mich, ausgekochtes trockenes Fleisch ziemlich schwach angeregt. Rohes Fleisch dagegen und besonders die Extraktivstoffe des Fleisches erregen die Tätigkeit der Magendrüsen im höchsten Grade, und zwar besonders die Sekretion der Magensaftsäure, welche wohl zu den schwersten chemischen Arbeiten des Protoplasmas gehört. Und diese Säure bewirkt wieder durch die Duodenalschleimhaut eine Erregung der Pankreassekretion.

Auf Grund dieser Tatsachen erhebe ich also meinen Gegeneinwand: die grosse Harnsäurevermehrung nach Fleisch- oder Bouilloneinnahme rührt von der sehr intensiven Tätigkeit der Verdauungsdrüsen her, welche gerade durch rliese Stoffe iu viel höherem Grade angeregt wird als durch die blossen Proteine der Milch-, Eier- und Mehlspeisen. Wenn behauptet wird, dass jene Vermehrung nur von den Purinstoffen des Fleisches als direkten

1) Pawlow, Nagel's Handb. d. Physiol. Bu. 2 (2) S, 666. 1907. 
Muttersubstanzen der Harnsäure im Organismus herrührt, so ist dafür ein direkter Beweis zu erbringen, in welchem alle Möglichkeit jener endlogenen Quelle der Harnsäure in der durch die Fleischaufnahme angeregten Tätigkeit der Verdauungsdrüsen ausgeschlossen wäre.

$\mathrm{Zu}$ einem solchen Beweise könnten vielleicht die von $\mathrm{Burian}$ und Schur ermittelten quantitativen Verhältnisse zwischen den aufgenommenen Purinbasen und der darauf folgenden Harnpurinvermehrung zugezogen werden. Danach ${ }^{1}$ ) gelangt nach Einnahme von Hypoxanthin und Xanthin etwa die Hälfte des aufgenommenen Purinstickstoffes in der Form von Harnsäure und Purinbasen durch den Harn zur Ausscheidung, nach Einnahme von Thymusnuklein und Adenin nur ein Viertel. Nach Zulage von Fleisch zur gewöhnlichen purinfreien Kost erscheint nun auch von dem im Fleische enthaltener Gesamtnahrungspurin- $\mathrm{N}$ etwa die Hälfte als Harnpurin- $\mathrm{N}$ wieder, als wären die darin enthaltenen Puringruppen einfach als Hypoxanthin verabreicht worden. Wenn also, wie wohl anzunehmen ist, nicht gerade das Hypoxanthin die Tätigkeit der Verdauungsdrüsen anregt, so ist die Harnsäurevermehrung nach Fleischeinnahme ganz direkt mit dessen Hypoxanthin in Zusammenhang zu bringen. Das Ergebnis wäre bindend, liesse die Versuchsanordnung Burian und Schur's nicht den oben angeführten Einwand zu, dass $Z$ ulagen von Fleisch zur gewöhnlichen, wenn auch purinfreien Kost in der Frage der endogenen Harnsäurebildung nicht entscheidend sind. Die in dieser Richtung berechneten Ergebnisse der mit der Methode des nüchternen Zustandes ausgeführten Versuche von $\mathrm{Hopkins}$ und $\mathrm{Hope}^{2}$ ) würden allerdings für den Standpunkt Burian und Schur's sprechen; so z. B. im Versuche I zeigte sich, nach Einnahme von $250 \mathrm{~g}$ Fleisch zu einer bestimmten Stunde des nüchternen Zustandes, in den folgenden 9 Stunden die Harnsăure gegenüber dem nüchternen Zustande um $0,133 \mathrm{~g}=0,044 \mathrm{~g} \mathrm{~N}$ vermehrt; mit dem Fleische wurden aber $0,15 \mathrm{~g}$ Purinstickstoff aufgenommen, wovon also $29 \%$ in Form von Harnsäure zur Ausscheidung kamen. In denselben Versuchen zeigte sich auch, dass die Harnsäurevernehrung mit der Menge des aufgenommenen Fleisches grösser wurde; nach Einnahme von $200 \mathrm{~g}$

1) Burian und Schur, Pflüger's Arch. Bd. 80 S. 315.

2) Hopkins and Hope, The Journ. of Physiol. vol. 23 p. 275. 
Fleisch betrug dieselbe $86 \mathrm{mg}$, nach $250 \mathrm{~g}-133 \mathrm{mg}$, nach $280 \mathrm{~g}-182 \mathrm{mg}$.

Es ist also wohl gar nicht in Abrede zu stellen, dass die Nahrungspurine zum Teile direkt in Harnpurine übergehen. Zum grösseren Teile werden dieselben aber doch im Säugetierorganismus weiter zersetzt [zu Allantoin ${ }^{1}$ )]. Nach Burian und Sebur ${ }^{2}$ ) bat der Fleischfresser das grösste Vermögen, Nahrungspurine zu zerstören, indem bei demselben nur der 20. Teil der aufgenommenen Nahrungspurine als Harnpurine zur Ausseheidung gelangt, beim Kaninchen der 6. Teil, beim Menschen aber die Hälfte, so dass der Mensch das geringste Vermögen besitzt, Nahrungspurine zu zerstören.

Wenn also uicht geleugnet werden kann, dass Nahrungspurine zum Teile direkt in Harnpurine übergehen, so kann andererseits auch nicht ausgeschlossen werden, dass purinhaltige Nahrung, welche erwiesenermaassen die Tätigkeit der Verdauungsdrùsen im höcbsten Grade anregt, auch diese end ogen e Quelle der Harnsäure eröffnet, so dass die Harnsäurevermehrung nach Fleischgenuss von diesen beiden Quellen herrühren kann, wobei auch das Purinzerstörungsvermögen des Organismus regulierend eingreifen könnte.

Es eröffnen sich hier Aussichtspunkte in die Physiologie und die Hygiene der Ernährung, deren allgemeine Bedeutung ich beiläufig so andeuten möchte. Die natürlichen Nahrungsmittel, Milch, Eier, Körner und Frächte, vorbereitet zur Ernährung der Nachkommenschaft, sind nuklein- und purinfrei, ihre Verdauung und Assimilation erfordert nicht viel Arbeit des Verdauungsorgans und verursacht nur geringe Vermehrung intermediärer Stoffwechselprodukte, wie der Harnsäure und der Harnpurine. Fleisch, Drusen usw. sind dagegen Organe, deren natiurliche Bestimmung eine ganz andere ist als als Nahrungsmittel zu dienen. Ihre Verdauung und Assimilation erfordert eine viel grössere Anstrengung des Verdauungsorgans, welche sie auch tatsächlich hervorrufen; ihre $\mathrm{Nu}$ kleine sind überhaupt schwer verdaulich, ihre Purinkörper schwer zerstörbar, so dass durch dieselben die eigenen endogenen Purinkörper vermehrt werden. Der auf diese Nahrung angepasste Fleischfresser hat auch ein starkes Vermögen, diese Purinkörper zu

1) W. Wiechowski, Biochem. Zeitschr. Bd. 25 \$. 431. 1910.

2) Burian und Schur, Pflüger's Arch. f. d. ges. Physiol. Bi. 87 S. 335. 1901. 
zerstören; der Mensch aber zeigt sich am schwächsten in dieser Beziehung, so dass Fleischnahrung für ihn nicht angemessen zu sein scheint. Die Überschätzung des Fleisches als Nahrungsmittel geschah aus dem Grunde, weil es im allgemeinen mit Protein inentifiziert wurde; dieses Missverständnis beherrschte lange Zeit die Physiologie der Ernährung, wovon ich ein eigenes Beispiel vorgefuhrt habe, und wovon viele berühmtere Beispiele angeführt werden könnten. Es hat wohl zuerst Sivén ${ }^{1}$ ) mit Nachdruck hervorgehoben, dass "Eiweiss" und "Fleisch" nicht als Synonyma angewandt werden dürfen. Die Proteine der natürlichen Nahrungsmittel, wie der Milch, des Eies und des Mehls, erscheinen auch in manch anderer Beziehung zur Ernährung des Menschen geeigneter als die Proteine der Muskeln und der anderen Körperorgane. Die Unterscheidung ist offenbar eine ganz andere als die zwischen animaler und vegetabilischer Nahrung.

Kehren wir nun zu unserer Hauptfrage zurück. Aus den zwei Versuchen Hopkins und Hope's ist also zu entnebmen, dass auch purinfreies Eiweiss eine deutliche Harnsäurevermebrung bewirkt. Aber selbst die Hauptvertreter der Ansicht; dass purinfreie Nahrung von keinem Einfluss auf die Harnsäureausscheidung sei, geben dennoch diesen Einfluss selbst zu, erklären denselben aber auf Umwegen. Burian und Schur ${ }^{2}$ ) erkennen z. B. an, dass im Hungerzustande die endogene Harnpurinbildung geringer ist als nach Genuss purinfreier und überhaupt stickstofffreier Kost, da im Hungerzustande „so wie zahlreiche andere Stoffwechselvorgänge auth die Bildungsprozesse der endogenen Harnpurine eingeschränkt werden". Und Sivén ${ }^{3}$ ) bewies den Einfluss purinfreier Eiweisskost auf die Harnsäureausscheidung auf folgende einwandfreie Weise. Während der Nachtruhe ist die Harnstickstoff- und Harnsäureausscheidung geringer als bei Tage, denn auch die Nieren ruhen während des Schlafes. Um die Nieren während des Schlafes zu erhöhter Arbeit anzureizen und zu sehen, ob dadurch die Harnsäureausscheidung vermehrt wird, verabreichte Si vén der Versuchsperson abends reichliche purinfreie Eiweisskost (Eier und Käse), um die Stickstoffausscheidung während der Nacht $z u$ steigern. Tatsäch-

1) V. O. Sivén, Skand. Arch. f. Physiol. Bd. 11 S. 135.1901.

2) Burian und Schur, Pflüger's Archiv f. d. ges. Physiol. Bd. 80 S. 275.

3) V. O. Sivén, Skand. Arch. f. Physiol. Bd. 18 S. 191. 1906. 
lich gluckte dies: die endogene Purinausscheidung während des Schlafes zeigte sich vermehrt. Werden also, so schliesst Sivén, die Nieren auf solche Weise während des Schlafes zu erhöhter Arbeit der Stickstoffausscheidung gezwungen, so steigt auch die Purinproduktion; und zwar, nach Sivén, in den Nieren. "Dieses kann nicht auf dem Eiweissgehalt der Kost an und für sich beruhen, denn, wie wir wissen, spielt derselbe beim Purinumsatz im Organismus keine Rolle, sondern wir müssen sie wohl mit der erhöhten Nierentätigkeit in Zusaummenhang bringen."

Wie wir wissen! Aber gerade dieser Versuch würde ja beweisen, dass der Fiweiss gehalt der Kost beim Purinumsatz eine Rolle spielt; und zwar, nach Sivén's Ansicht, in der Weise, dass die dadurch vermehrte Harnstoffbildung die Nieren zur grösseren Ausscheidungsarbeit zwingen würde, welche mit Harnsäurebildung verbunden wäre. Wir wissen aber, dass die Menge des Harnstickstoffes, welche von dem Eiweissgehalt der Nahrung abhängig ist, in keinem Verhältnis zur Harnsäuremenge steht; dieser Satz, an dessen Feststellung Sivén selbst hervorragend beteiligt war, schliesst aus, dass vermehrte Harnstickstoffausscheidung vermehrte Harnsäurebildung durch erhöhte Nierentätigkeit bewirken würde; dieses wird auch durch die festgestellte Tatsache ausgeschlossen, dass die Vermehrung des Harnstickstoffes nach rejchlicher Eiweisskost später eintritt und länger andauert als die Vermehrung der Harnsäure, welche sogleich nach der Nahrungsaufnahme anhebt und in einigen Stunden schwindet. Der Versuch Sivén's beweist aber ganz gewiss, dass purinfreie Eiweisskost Harnsäurevermehrung bewirkt; und darauf kommt es uns au. Wenn es nicht durch Anregung der Nierentätigkeit gesehieht, so kann es durch Anregung der Tätigkeit anderer Drüsen geschehen, welche früher eintreten muss, als es zur Verlauung des Nahrungseiweisses und zur Bildung und Ausscheidung des Harnstoffes kommt. Die Nieren verrichten bei der Harnstoffausscheidung doch nur eine mechanische Arbeit, indem sie fertige Stoftwechselprodukte aus dem Blute ausscheiden. Die Verdauungsdrüsen aber siud auf Anregung der Nahrung chemisch tätig, indem sie aus ihrem eigenen Zelleibe Sekrete bilden, woran die Zellkerne sichtbar beteiligt sind; hier können Nukleinschlacken entstehen.

Die Position der Vertreter des "Satzes, tass purinfreie Eiweissnahrung keinen Einfluss auf die Bildung endogener Harnsäure 
habe, findet auch $\mathrm{O}$. Folin ${ }^{1}$ ) nicht besonders gut befestigt; die Versuche von Burian und Schur sowie die von Sivén seien nicht genügend zum Beweise des fraglichen Satzes. Wenn tatsächlich der Gesamtwert des Proteinmetabolismus sehr vermindert wird, so werde auch die Menge der Harnsäure vermindert, allerdings nicht in gleichem Maasse wie der Harnstickstoff, so dass der Anteil der Harnsäure an diesem steigt. Die Harnsäureverminderung bei hochgradiger Herabsetzung des Proteinumsatzes fand Folin unregelmässig und verschieden bei verschiedenen Versuchspersonen und bezeichnete demnach den harnsäurebildenden Metabolismus als relativ instabil; die Harnsäure zeige unter diesen Umständen nicht die charakteristische quantitative Konstanz, wie das Kreatinin. Sollte die endogene Harnsäure, sagt Folin, als von den Zellnukleinen berruhhrend betrachtet werden, so würde ihre Menge sehr wahrscheinlich konstant bleiben auch bei sehr grossen Variationen der Diät; ein strenger Beweis, dass die Harnsäure für ein jedes Individuum einen konstanten Wert besitzt, würde ein starkes Zeugnis für diese Theorie sein.

Nach Folin also ist der Einfluss purinfreier Eiweisskost auf die endogene Harnsäurebildung unzweifelhaft. Ist dem aber so, wie kann die individuelle Konstanz des Harnsäurewertes bei sehr grossen Variationen der Diät hervortreten? Tatsächlich babe ich die individuelle Konstanz der Harnsäure im nüchternen $\mathrm{Zu}$ stande nachgewiesen, wo besonders die Verdauungstätigkeit ruht und der Organismus von seinen Reserven lebt. Der Einwand Folin's gilt dagegen den Versuchen Burian und Schur's gegenüber, welche die individuelle Konstanz der Harnsäure bei regelmăssiger Einnahme purinfreier Kost bestimmten, unter der Voraussetzung, dass diese Kost keinen Einfluss auf die endogene Harnsäurebildung ausübt. Der $\mathrm{Nachweis}$ der individuellen Konstanz der Harnsäure gilt also nur fur den nüchternen Zustand. Auch $B u_{i}$ rian ${ }^{2}$ ) ist zuletzt zu dieser Ansicht gekommen: im Hungerzustand fällt die störende Einwirkung der Nahrungsaufnahme weg, und die Harnsäure stellt sich etwa von der 15. Hungerstunde ab auf ein fast ganz konstantes Niveau ein (Hungerstandard der Harnpurinausfubr).

1) 0. Folin, The Amer. Journ. of Physiol. vol. 13 p. 66. 1905.

2) R. Burian; Zeitschr. f. physiol. Chemie Bd. 43 S. 534 . 1904-1905. 
Die von mir ausgesprochene Theorie, dass die (endogene) Harnsäure von den Zellnukleinen herrührt, ist also durch das Zeugnis der individuellen Konstanz derselben im $\mathrm{nüchternen}$ Zustande, gemäss der Forderung Folin's, stark befestigt; und die Tatsache, dass dureh Aufnahme auch purinfreier Nahrung Harnsäurevermehrung bewirkt wird, zeugt dafur, dass hierbei die Zellkerne der Verdauungsdrüsen wirksam sind, wofür auch der zeitliche Vrrlauf der Harnsäurevermehrung spricht.

Die Beziehungen der endogenen Harnsäure zur Verdauung sind in neuerer Zeit von Hirschstein ${ }^{1}$ ) (Umber) untersucht worden, ausgehend von dem festen Punkte, dass der endogene Harnsäurewert bei purinfreier Kost $g \mathrm{r} o ̈ s s$ er ist als der Hungerwert $(0,381 \mathrm{~g}-0,181 \mathrm{~g})$. Die Verdauungstätigkeit hat demnach unzweifelhaft den grössten Einfluss auf die Ausscheidung endogener Harnsäure; wird die Hauptmahlzeit verschoben, so verschiebt sich auch die Harnsäureausscheidung; reichliche purinfreie Eiweissnahrung steigert die endogene Harnsäure um 6-10\%; diese hat zu einem hohen Prozentsatz $(70 \%)$ ihren Ursprung in der Verdauungstätigkeit. In Darminhalte eines Hundes, der 3-4 Stunden nach Verabreichung reichlicher purinfreier Eiweissnabrung getötet wurde, fand sich eine grosse Menge von Purinstoffen (besonders Guanin): „Das eine geht aus unseren Versuchen unzweifelhaft hervor, dass während der Verdauung die dem Digestionstraktus zugehörenden Drüsen ein purinhaltiges Sekret liefern. Daraus ist der Schluss wahrscheinlich, dass auch beim Menschen die während der Verdauungstätigkeit ausgeschiedene Harnsäure diesen Purinkörpern ihren Ursprung verdankt".

Der Zusammenhang der endogenen Harnsäurebildung mit den Verdauungsdrüsen tritt besonders beim Pankreas hervor. Es wäre hier zunächst die auffallend geringe Ausscheidung der endogenen Harnsäure bei Pankreas-Erkrankungen zu erwähnen, welche von Bloch und Rosenberger ${ }^{2}$ ) beobachtet wurde; nach purinarmer Nahrung war die Harnsäureausscheidung sehr verringert, während nach purinreicher Kost dieselbe beträchtlich vermehrt war. Bloch schloss auf eine Alteration des Purinstoffwechsels beim Diabetes; Rosenberger fand, dass die in Frage stehenden Kranken exogene

1) L. Hirschstein, Arch. f. experim. Path. und Pharm. Bd. 57 S. 229. 1907.

2) F. Rosenberger, Zeitsrhr. f. Biol. Bd. 48 S. 529.1906. 
Purine prompt ausscheideu, ihre endogenen Werte aber nieder sind. Dieses Verhältnis würde sich aufklären, wenn gerade das Pankreas, im gesunden Zustande, eine der Hauptquellen der endogenen Harnsäure wäre. In der Tat fand de $\mathrm{Zilva}{ }^{\mathrm{J}}$ ) das Pankreassekret des Hundes, welches durch Anregung der Drüsentätigkeit durch Pilokarpin gewonnen wurde, sehr reich an Nukleinsubstanzen, was auf einen intensiven Nuklein-Metabolismus in der tätigen Drüse hindeutet.

So gelangen wir zum Pilokarpinversuch, welchen ich zuerst zur Begründung des Zusammenhanges der Harnsäurebildung mit der Drüsentätigkeit projektiert und durchgeführt habe. Durch den Nachweis einer Harnsäurevermehrung nach Pilokarpininjektion glaubte ich die Kette der Beweisführung geschlossen zu haben; aber auch darin wurde ich getäuscht. Ich übersah, dass das Pilokarpin nicht nur die Drüsen zur Tätigkeit anregt, sondern auch die glatten Muskeln zur Kontraktion bringt; und durch diese Lucke entschlüpfte mir wieder der Erfolg.

Die Tatsache, dass durch Pilokarpin eine deutliche Harnsäurevermehrung bewirkt wird, ist zunächst von Horbaczewski²) bestätigt worden, dem aber dafür „bis nunzu keine ausreichende Erklärung" getunden zu sein schien, so dass es ihm von grossem Interesse war, vor allem das Verhalten des Leuk ocy tengehal tes des Blutes unter der Einwirkung dieses und anderer Gifte zu prüfen. Wirklich fand er nach Pilokarpineinwirkung eine entschiedene Vermehrung des Leukocytengehaltes des Blutes; es wurden auch karyokinetische Veränderungen in der Pilokarpiumilz beobachtet, welche grösser als "normal" gefunden wurde, obzwar das Pilokarpin eiue starke Darmkontraktion bewirkt hatte. Und so ist mein Pilokarpinversuch zu einer Stütze der Leukocy tosetheorie geworden.

Aber diese Theorie hat sich schliesslich auch in diesem Punkte nicht bewährt. Horbaczewski selbst hatte hier ein gewisses Missverhältnis zwischen der Grösse der Leukocytose und der. Grösse der sie begleitenden Harnsäureausscheidung bemerkt, da nach Pilokarpin sowie Nuklein eine relativ sehr intensive Leukocytose von einer viel geringeren Harusäurevermehrung begleitet wird als nach

1) L. de Zilva, The Journ. of Physiol. vol. 31 p. 230.1904.

2) J. Horbaczewski, Sitzungsber. der kais. Akad. d. Wissensch. Wien Bd. 100 Abt. III S. 25. April 1891. 
Aufnahme grosser Fleischmengen. Hopkin's und Hope haben deun auch dieses Missverhältnis gegen die Leukocytosetheorie hervorgehoben. Nun zeigt sich, dass die Pilokarpinleukocytose gar nicht auf einer materiellen Vermehrung der Leukocyten „durch Alteration der lymphatischen Organe, die die Leukocyten produzieren", beruht, sondern dass sie rein mechanisch ist, entstanden durcb Kontraktion der glatten Milzmuskeln; und dass somit auch die Harnsäurevermehrung durch Pilokarpin mit dieser mechanischen Leukocytose in keinem Zusammenhange steht. Harvey ${ }^{1}$ ) hat gezeigt, dass die Pilokarpinleukocytose nicht zustande kommt, wenu der Einwirkung des Pilokarpins auf die glatten Milzmuskeln durch Atropinisierung vorgebeugt wird, oder wenn die Milzgefässe unterbunden werden; und ebensolche Leukocytose entsteht auch durch Kontraktion der Milzmuskeln nach Reizung der Milznerven.

Indessen findet die Leukocytosetheorie noch immer neue Vertreter. Vor zehn Jahren war diese Theorie vorherrschend und diente als Ausgangspunkt verschiedener Untersuchungen, welche manchmal zu ganz sonderbaren Ergebnissen geführt haben. So hatte z. B. A. Matthews ${ }^{2}$ ) zwischen der Menge des Fibrinogens im Blute und der Harnsäureausscheidung einen engen Parallelismus beobachtet, welcher auf ihren gemeinschaftlichen Ursprung in dem Zerfalle der Blutleukocyten zurückzuführen sei; und zwar rühre die Harnsäure vom Nuklein der Leukocyten her, das Fibrinogen aber aus dem Zytoplasma derselben: „in the cytoplasm of the cells it will be remembered that the reticulum takes a fibrillar form, these fibrils strongly reminding of the fibrinfibrils".

Aber es kam noch Besseres, jetzt, wo die Theorie eigentlich schon aufgegeben ist. Wie hier nie Fibrinfibrillen durch das Fibrinogen hindurch als Cytoplasmafibrillen hindurchschimmern, so repräsentieren wieder die Leukocyten des Säugetierblutes eigentlich wirbellose Tiere. Plimmer, Dick und $\mathrm{Li} \mathrm{eb}^{3}$ ) haben das feste Verhältnis zwischen der Leukocytenzahl des Blutes und der ausgeschiedenen Harnsäuremenge von neuem bewiesen, da dies von den meisten neueren Untersuchern vernachlässigt wurde. Sie fanden einen ganz konstanten Parallelismus zwischen der Leukocytenzabl

1) W. H. Harvey, The Journ. of Physiol. vol. 35 p. 115. 1906.

2) A. Matthews, The Amer. Journ. of Physiol. vol. 3 p. 78. 1899.

3) Plimmer, Dick, Lieb, The Journ. of Physiol, vol. 39 p. 98, 1909. 
im Blute und der ausgeschiedenen Harnsäuremenge; dagegen aber gar keine Beziehung zwischen dieser und den eingenommenen Purinen. Deshalb halten Sie nur die aus den Nukleinen der Leukocyten entstehenden Purine für Mutterstoffe der Harnsäure. "In mammals the endproduct (of the foodmetabolism) is urea... in many invertebrates it is uric acid. In leukocytes, as invertebrates, the endproduct of metabolism is most probably uric acid." Die Leukocytosetheorie trägt also noch immer ihre eigentümlichen Früchte; hier lässt sie z. B. in den Drüsenzellen der Wirbeltiere eine Wirbelsäule hindurchschimmern, wogegen ihre Leukocyten wirbellos wären.

Die Harnsäurevermehrung nach Pilokarpineinwirkung hängt also zweifellos mit der dadurch angeregten Drüsentätigkeit zusammen; für das Pankreas ist dieser Zusammenhang durch den angefulurten Versuch de Zilva's nahezu direkt dargetan. Mein Pilokarpinversuch ist also in dem Sinne zu deuten, in welchem ich denselben zuerst entworfen und durchgeführt habe: die Drüsentätigkeit ist mit Harnsäurebild ung verbunden.

Um diesem Satze eine noch breitere tatsächliche Grundlage zu geben, dachte ich daran, die Tätigkeit der Verdauungsdrüsen, ausser durch purinfreie Nahrungsstoffe, auch auf eine canz immaterielle Weise anzuregen, nämlich durch Erregung einer „psychischen Sekretion" nach Pawlow. Zu diesem Zwecke sollte vor der im nüchternen Zustande sich befindenden Versuchsperson ein anderer ein leckeres Mahl einnehmen, wobei sich dieselbe dem so erregten "Appetite" womöglich ganz hingeben sollte. Diese Versuche blieben vorläufig, in bezug auf die Harnsäureausscheidung, ohne ein entschiedenes Ergebnis. Vielleicht ist die auf solche Weise erregte Drüsentätigkeit nicht intensiv genug, indem sie auf die Speicheldrüsen beschränkt bleibt. Popielski ${ }^{1}$ ) behauptet gegen Pawlow, dass die Magendrüsen überhaupt nicht „psyehisch" erregbar sind, sondern nur durch einen gewöhnlichen Reflex, hervorgerufen durch mechanische Reizung des Zahnfleisches beim Kauen. Das Pankreas dürfte auf solche Weise überhaupt nicht in Tätigkeit zu bringen sein. Nichtsdestoweniger würde die durch „kortikale Reflexe" hervorgerufene Drüsentätigkeit und ihr Verhältnis zur HarnsäureAusscheidung noch weiterer Untersuchungen wert sein, 


\section{Schlussbetrachtungen.}

Die ersten Forscher, welche sich in den verflossenen zwanzig Jahren mit der Frage der Herkunft der Harnpurine und besonders der Harnsäure beim Menschen führend beschäftigt haben, bezeugen, dass durch diese überaus zahlreichen Untersuchungen die Frage bisher noch nicht in befriedigender Weise beantwortet ist. So hat sich Burian ${ }^{1}$ ) ausgesprochen und suchte in der Muskeltätigkeit eine Quelle der endogenen Harnpurine. Sivén ${ }^{2}$ ) aber, welcher an der Stelle der abgeschafften Leukocytosetheorie keine andere wahrscheinlichere gefunden hat, weil er die Herkunft der Harnsäure aus dem Stoffwechsel der Verdaunngsdrüsen ausschliessen zu können glaubte, betrachtete auch sehr skeptisch Burian's Versuch über die Muskelquelle der Harnpurine, obgleich er selbst zuerst diese Möglichkeit ausgesprochen hatte, und suchte in der $\mathrm{Nier}$ en tätigkeit die endogene Quelle der Harnsäure.

Eine solche Divergenz der Forschungsrichtungen zeigt am deutlichsten den gänzlichen Mangel eines einheitlichen GesichtsPunktes oder e in er T h e orie der Harnsäurebildung beim Menschen. Daraus schöpfte ich die Berechtigung sowie die Aufforderung, meine ursprungliche Theorie von neuem darzulegen und die gegen dieselbe erhobenen Einwände kritisch zu beleuchten und zu zerstreuen. Und ich glaube, dass es mir tatsächlich gelungen ist. Der Fortschritt, welchen zwanzigjährige rastlose Forschungen auf diesem Gebiete gebracht haben, mag gering erscheinen, wenn es möglich ist ältere und anfangs wenig beachtete Gesichtspunkte von neuem einzuführen; vielleicht besteht aber dieser Fortschritt eben darin, dass dies gegenwärtig mit mehr Aussicht auf Beachtung und Erfolg geschehen kann als vor 23 Jahren.

Der allgemeine Satz, dass die Harnsäure, und die Harnpurine überhaupt, ein Produkt des physiologischen Stoffwechsels der Körperzellen ist, woran besonders die Zellkerne beteiligt sind, wird auch fast allgemein angenommen. Aber der Ursprung und die Beglaubigung dieses Satzes sind den meisten ziemlich dunkel. S i vén ${ }^{3}$ ) führte diesen Satz auf Kossel zurück; weil dieser den chemischen Zusammenhang der Alloxurbasen mit den Nukleinen aufgedeckt

1) R. Burian, Zeitschr. f. physiol. Chemie Bd. 43 S. 532 . 1904-1905.

2) O. Sivén, Skand. Arch. f. Physiol. Bd. 18 S. 189. 1906.

3). O. Sivén, Skand. Arch. f. Physiol, Bl. 11 S. 146. 
hatte. Aber diese chemische Tatsache wäre für sich allein eïn doch zu schmaler Grund für einen so breiten physiologischen Satz. Andere deuten auf Horbaczewski hin, weil er den chemischen Zusammenhang der Harnsäure mit den Alloxurkörpern und den Nukleinen erwiesen hatte. Ellinger ${ }^{\text {) }}$ sagt z. B.: ${ }_{\text {Die }}$ bestehende Lehre von der Bildung und Ausscheidung der Harnsäure beruht durchaus auf den von Horbaczewski ermittelten Tatsachen, aber der Entdecker selbst hat die Entwickelung dieser Lehre in eine Sackgasse geführt ...."

Wahrlich gerieten hier in eine Sackgasse nur die Physiologen, welche nicht erkannten, dass: zur Begründung einer physiologischen Theorie blosse chemische Tatsachen nicht hinreichen. Die Tatsache, dass die Purinkörper mit den Nukleinen ehemisch zusammenhängen, und der Satz, dass die Harnpurine durch den physiologischen Stoffwechsel in den Zellkernen entstehen, sind gar nicht gleichbedeutend; zu diesem Satze sind nebst jener chemischen Tatsache atch noch physiolog ische Tatsachen notwendig. Auf einer bloss chemischen Tatsache kann eine bloss chemische Theorie aufgebaut werden; und eine solche war auch die Leukocytosetheorie von Horbaczewski: ebenso wie bei der Fäulnis der Milzpulpa entsteht auch im lebenden Körper die Harnsäure aus dem Nuklein der nekrotisch zerfallenen Leukocyten. Nach dieser Anschauung spielt dieses Nuklein bei der Harnsäurebildung ganz dieselbe Rolle, wie das mit der Nahrung eingenommene Nuklein, so dass die Harnsäurebildung ganz ausserhalb des eigentlichen physiologischen Stoffwechsels, also uur exogen vor sich gehen würde: Und wirklich, diese Wendung nabm deun auch die Frage des Harnsänreursprungs. Es gebührt Sivén und Burian mit Sch u r das Verdienst, diese Frage wieder auf p h y s i ologise be n Boden gebracht zu haben, durch Feststellung und Nachweis des endogenen Anteils der Harnpurine. Die Physiologen besannen sich endlich auf ihre eigentliche Aufgabe, und das war der logische Grund des vollständigen Niederganges der Leukocytosetheorie.

Die physiologische Theorie, nach welcher die Harppurine. durch den Stoffwechsel der lebenden und tätigen Zellen (Protoplasma) entstehen, muss auf ph y si $010 \mathrm{~g}$ i s chen Tatsachen beruhen;

1) A. Ellinger, Handb. d. Biochemie, herausgeg. von oppenheimer, Bd. 3 (1) S. 574.1909. 
die chemische Erkenttnis des Zusammenhanges zwisehen den Purinkörpern und den Nukleinen ist dabei gewiss sehr wichtig, aber doch nur nebensächlich, indem dadurch eine nähere Bestimmung jenes Stoffwechsels möglich wird, in dem Sinne, dass an demselben die Zellkerne beteiligt sein dürften, was aber erst weiter physiologisch festzustellen wäre.

Die physiologische tatsächliche Grundlage des Satzes, dass die Harnsäure ein Produkt des physiologischen Stoffwechsels im Protoplasma der Körperzellen darstellt, ist von mir auch vor der Erkenntnis des Zusammenhanges der Harnsäure mit den Nukleinen festgelegt worden, und ich babe auch nach dieser Erkenntnis den Satz in dem Sinne erweitert, dass an jenem Stoffwechsel die Z ellkerne beteiligt sind.

Jene tatsächliche Grundlage war die folgende:

1. Individuelle Konstanz der Harnsäuremenge bei verschiedenen Personen in einer bestimmten Periode des nüchternen Zustandes, im Gegensatze zur grossen Veränderlichkeit des gesamten Harnstickstoffes (Harnstoffes), welche von dem Eiweissgehalte der zuletzt eingenommenen Nahrung abhängig ist.

Diese Tatsache weist darauf hin, dass die Harnsäurebildung von einem individuellen Faktor abhänøig ist, als welcher im allgemeinen das Protoplasma der Körperzellen zu bezeichnen wäre, insoferne dasselbe bei seinen physiologischen Verrichtungen einem Stoffwechsel unterliegt.

Dieser Schluss ist einwandlos. Auch Folin ${ }^{1}$ ), der die Tatsache der individuellen Konstanz der Harnsäuremenge bezweifelt und dafür eine solche des Kreatinins annimmt, hält einen genauen Nachweis dieser Tatsache für ein starkes Zeugnis zugunsten dieser Theorie.

2. Mit der Steigerung der physiologischen Tätigkeit der Körperzellen, soferne dieselbe mit stofflichen Änderungen im Protoplasma verbunden ist, steigt auch die (endogene) Harnsäurebildung.

Diese Tatsache ist im allgemeinen beim rasch wachsenden Organismus, besonders beim Neugeborenen zu bemerken, wo eine rasche karyokinetische Zellenvermehrung anzunehmen ist.

Besonders aber tritt diese Tatsache bei Anregung der Tätigkeit der Verdauungsdrüsen hervor, wobei eingreifende stoffliche

1) O. Folin; Amer. Journ. of Physiol. vol. 13 p. 88.1905. 
Änderungen im Protoplasma der Drüsenzellen zu sehen sind, an welchen besonders auch die Zellkerne sich beteiligen.

Diese Anregung geschieht auf natürliche Weise durch die eingenommene Nahrung, in verschiedenem Grade, je nach der Qualität dieser Nahrung. Danach tritt eine Steigerung der Harnsäureausscheidung ein, welche ibrem zeitlichen Verlaufe nach mit der Tätigkeit der Verdaungsdrüsen einhergeht, wogegen die mit Eiweissnahrung verbundene Harnstickstoffvermehrung später eintritt und viel länger andauert.

Der Einwand, dass diese Harnsäurevermehrung von den Purinstoffen der Nahrung herrübrt, wird durch die Tatsache entkräftet, dass auch purinfreie Nahrung eine solche, wenn auch geringere Harnsäurevermehrung bewirkt. Er kann auch durch den Gegeneinwand geschwächt werden, dass gerade die purinreichen Nalırungsstoffe die Tätigkeit der Verdauungsdrüsen im höchsten Grade in Anspruch nehmen und auch anregen.

Endlich ist auch die künstlich durch das Pilokarpin angeregte Tätigkeit der Verdauungsdrüsen mit einer deutlichen und zeitlich entsprechenden Harnsäurevermehrung verbunden. Dazu kommt, dass tatsächlich bei dieser Tätigkeit, wenigstens beim Pankreas, ein nukleinhaltiges Sekret geliefert wird, was einen intensiven Nukleinmetabolismus bei der Tätigkeit dieser Drüse beweist.

Durch dies alles erscheint der Nachweis gelungen, dass die Zellen der Verdauungsdrüsen durch ihren physiologischen Stoffwechsel Harnsäure produzieren. Und dieser Nachweis bildet die tatsächliche physiologische Grundlage des allgemeinen Satzes, dass die Harnsäure ein Produkt des physiologischen Stoffwechsels in den Körperzellen ist, an welchem besonders die Zellkerne beteiligt sind. Ich ging wirklich so weit, wie Burian und $\mathrm{Schur}{ }^{1}$ ) bemerkt habev, in der ausgeschiedenen Harnsäuremenge ein Maass nicht des "Protoplasmazerfalles",-, sondern des phy si ologi s c he n St off wechsels im "Protoplasma" zu sehen.

Der angeführte allgemeine Satz bedeutet eine Theorie der. Harnsäurebildung im menschlichen Organismus; das ist, derselbe fürt zu Folgerungen, welche als Fragen für weitere Untersuchungen von Bedeutung sind. Die Güte einer Theorie richtet sich aber nach den Fragen, welche sie zu stellen weiss.

1) Burian und Schur, Pflüger's Arch. Bd. 80 S. 252. 
Eine jede Körperzelle, deren physiologische Verrichtungen mit stofflichen Änderungeu im Protoplasma und besonders in der Kernsubstanz verbunden sind, produziert Harnsäure, deren Melige ein Maass jener Änderungen abgeben könnte. Die allgemeinste Verrichtung dieser Art ist Ernährung, Vermehrung und Wachstum. Tatsächlich ist die Harnsäureproduktion beim rasch wachsenden Organismus überaus gross. Beim erwachsenen Organismus, dessen Verrichtungen sich allgemein in einem Gleichgewicht befinden, ist auch die Harnsäureproduktion ganz gleichmässig, individuell konstant. Das Verhalten der Harnsäureausscheidung im Greisenalter ist noch zu untersuchen ${ }^{1}$ ). Auch pathologische Zellwucherungen können mit Harnsäurevermehrung verbunden sein [Karzinom ${ }^{2}$ )].

Im besonderen können Zellen verschiedener Organe Harnsäure bilden. Neben den Drüsenzellen des Verdauungsapparates, vou welchen es als erwiesen gelten kann, ist an erster Stelle an die adenoiden Organe zu denken. Die Leukocyten sind zweifellos sehr aktive Harnsäureproduzenten, nicht durch ihren nekrotischen Zerfall, sondern durch ibre mannigfaltigen Verrichtungen, wie karyokinetische Vermehrung, intrazelluläre Verdauung, Cytasenbildung usw. Steigerung dieser Verrichtungen durch verschiedene, auch pathologische Einflüsse, ist tatsächlich mit Harnsäurevermehrung verbunden (myelogene Leukämie, Infektionskrankheiten und ähnliche).

Ganz besonders wären die Verhältnisse der Harnsäurebildung bei der Blutbildung der Säugetiere zu untersuchen. Grosse, akute Blutverluste regen die Blutkörperchenbildung im Knochenmarke intensiv an, wobei, nach Freytag ${ }^{3}$ ), sehr eingreifende Veränderungen in den Zellen, besonders in den Kernsubstanzen vor sich gehen. H. Strauss ${ }^{4}$ ) resumiert die Frgebnisse der Harnsäurebestimmungen nach akuten Blutverlusten beim Menschen in dem Sinne, dass in manchen Fällen die Purinausscheidung erhöht war. Es müssten darüber bestimmte physiologische Versuche angestellt werden.

1) Vgl. M. Levy, v. Noorden's Handb. der Pathol. des Stoffwechsels Bd. 1 S. 472.

2) Vgl. A. Schmidt, v. Noorden's Handb. der Pathol. des Stoffwechsels Bd. 2 S. 375.

3) Freytag, Zeitschr. f. allgem. Physiol. Bd. 8 S. 451. 1908.

4) H. Strauss, v. Noorden's Handb. der Pathol. des Stoffwechsels Bd. 1 S. 902.1906 . 
In neuerer Zeit ist von Burian ${ }^{1}$ ) die Muskeltätigkeit als eine ergiebige Quelle der endogenen Harnpurine bezeichnet worden, in Hinsicht darauf, dass im Stoffwechsel des lebenden Muskels Hурох anthin kontinuierlich gebildet wird. Lässt man, nach Burian, die Versuchsperson im nüchternen Zustande eine Stunde intensive Muskelarbeit leisten, so erfolgt hiernach eine stundenlang anhaltende bedeutende Steigerung der stündlichen Harnpurinwerte, anfangs hauptsächlich der Purinbasen, dann der Harnsäure, deren Maximum meist in der zweiten Stunde nach Ende der Arbeit erscheint, deren Wert aber später niedriger wird als vor der Arbeit. Durchströmungsversuche an überlebenden tetanisierten Hundemuskeln zeigten auch eine Vermehrung der Harnsäure.

Diese Versuche Burian's, von Sivén skeptisch beurteilt, fanden doch eine Bestätigung und Erweiterung durch die Untersuchungen von $\mathrm{Catheart,}$ Kennaway und Leathes ${ }^{2}$ ), welche während der Muskelarbeit eine Steigerung der Harnpurinbasen, aber eine Verminderung der Harnsäure, nach der Arbeit dagegen eine grosse und rapide Steigerung der Harnsäure beobachtet haben; daraus der Schluss, dass durch Muskelarbeit eine grosse Stejgerung der Purinbildung bewirkt wird, dass aber wegen des während der Arbeit herrschenden Sauerstoffmangels die Oxydation dieser Purine zu Harnsäure beschränkt ist, woraus sich die Wichtigkeit der gleichzeitigen Bestimmung der Harnsäure und der Harnpurine ergeben würde.

Der Finfluss intensiver und andauernder Muskelarbeit auf die Harnpurinbildung ist wohl nicht zu bezweifeln; aber die Muskeltätigkeit jst eine sehr komplizierte Verrichtung, so dass hier die eigentliche Purinquelle schwer zu bestimmen ist." Nach unserer Theorie entsteht die Harnsäure (und die Harnpurine) bei physiologischen Tätigkeiten der Zelle, welche mit stofflichen Änderungen im Protoplasma und Kern verbunden sind.: Die Muskeltätigkeit aber, sofern es sich um mechanische oder thermische Leistung handelt, braucht nicht direkt mit einem solchen Stoffumsatze in der Muske]substanz verbunden zu sein; die e ne rge ti s c b e Leistung des Muskels braucht nicht auf chemischer Grundlage zu ge-

1) R. Burian, Zeitschr. f. physiol. Chemie Bd. 43 S. 5832. 1904-1905.

2) Cathcart, Kennaway und Leathes, zit. Kennaway, The Journ. of Physiol. vol. 38 p. 1. 1908. 
102 F. Mareš: Der physiol. Protoplasmastoffwechsel und die Purinbildung.

schehen. Dagegen könnte wohl die Ernährung des Muskels während und besonders $\mathrm{nach}$ seiner energetischen Leistung mit einem Stoffumsatz im Sarkoplasma und seinen Kernsubstanzen verbunden sein, bei welchem Purinstoffe entstehen könnten. Tatsächlich gibt Kennaway an, dass eine bedeutende Harnsäurevermehrung nach intensiver Muskelarbeit bis 48 Stunden lang andauern kann. Zu dem Ernährungsstoffwechsel des Muskels gehört aber auch die glykogene Tätigkeit der Leber, welche als ein intrazellularer Verdauungsprozess mit Harnsäurebildung verbunden sein könnte. Übrigens bewirkt der thermische Effekt der Muskeltätigkeit intensive Schweisssekretion. Es ist also die Muskeltätigkeit mit der Tätigkeit mancher Drüsen verkoppelt, so dass diese endogene Purinquelle verschiedene Ursprünge haben kann.

Unserer Theorie gemäss ist eine Harnsäurevermehrung bei solchen Verrichtungen der Zellen zu erwarten, welche als eine chemische Arbeit betrachtet werden könnten. Das sind im allgemeinen alle physiologisehen Synthesen, deren Gesamtheit als Ernährung bezeichnet werden kann. Aber auch ganz einfache Synthesen könnten hierher gehören.

Zunächst die zuerst erkannte chemische Synthese im Säugetierkörper. Wenn die Niere fertigen Harnstoff aus dem Blute auszuscheiden hat, so ist diese eigentlich mechanische Verrichtung, wie ich Sivén gegenüber bemerkt habe, wohl nicht mit Harnsäurebildung verbunden. Es fragt sich aber, ob dies nicht der Fall ist, wenn die Niere aus Benzoesäure Hippursäure zu bilden hat?

Einnahme von neutralem Fett bewirkt, nach übereinstimmenden Angaben, eher eine Verminderung der Harnsäure. Aber Einnahme von Glycerin verursacht, nach $\mathrm{H}$ orbaczewski und Kanera, eine Vermehrung derselben. Es fragt sich, ob Einnahme von freien Fettsäuren oder Seifen, welche erwiesenermaassen im Körper zu neutralen Fetten ungebildet werden, eine Vermehrung der Harnsäure bewirkt?

Eine andere solche Frage ist, ob die Bildung von Glykogen in der Leber mit Harnsäurebildung verbunden ist. Diese Frage wird in einer nachfolgenden Abhandlung von Herrn S metánka beantwortet werden. 